\title{
Galaxy evolution in groups
}

\section{NGC 3447/NGC 3447A: the odd couple in LGG 225^}

\author{
P. Mazzei ${ }^{1}$, A. Marino ${ }^{1}$, R. Rampazzo ${ }^{1}$, H. Plana ${ }^{2}$, M. Rosado ${ }^{3}$, and L. Arias ${ }^{4}$ \\ 1 INAF Osservatorio Astronomico di Padova, Vicolo dell'Osservatorio 5, 35122 Padova, Italy \\ e-mail: paola.mazzei@oapd.inaf.it \\ 2 Laboratório de Astrofísica Teórica e Observational, Universidade Estadual de Santa Cruz, 45650-000 Ilhéus, Bahia, Brazil \\ 3 Instituto de Astronomía, Universidad Nacional Autónoma de México, Av. Universidad 3000, Ciudad Universitaria, C.P. 04510, \\ Mexico \\ 4 Universidad Iberoamericana, Department of Physics, México City, México D.F., Mexico
}

Received 17 May 2017 / Accepted 19 October 2017

\begin{abstract}
Context. Local Group (LG) analogs (LGAs) are galaxy associations dominated by a few bright spirals reminiscent of the LG. The NGC 3447/NGC 3447A system is a member of the LGG 225 group, a nearby LGA. This system is considered a physical pair composed of an intermediate-luminosity late-type spiral, NGC 3447 itself, and an irregular companion, NGC 3447A, linked by a faint, short filament of matter. A ring-like structure in the NGC 3447 outskirts has been emphasised by Galaxy Evolution Explorer (GALEX) observations.

Aims. This work aims to contribute to the study of galaxy evolution in low-density environments, a favourable habitat to highly effective encounters, shedding light on the evolution of the NGC 3447/NGC 3447A system.

Methods. We performed a multi- $\lambda$ analysis of the surface photometry of this system to derive its spectral energy distribution and structural properties using ultraviolet (UV), Swift UVOT, and optical Sloan Digital Sky Survey (SDSS) images complemented with available far-IR observations. We also characterised the velocity field of the pair using two-dimensional $\mathrm{H} \alpha$ kinematical observations of the system obtained with PUMA Fabry-Perot interferometer at the $2.1 \mathrm{~m}$ telescope of San Pedro Mártir (Mexico). All these data are used to constrain smooth particle hydrodynamic simulations with chemo-photometric implementation to shed light on the evolution of this system.

Results. The luminosity profiles, from UV to optical wavelengths, are all consistent with the presence of a disc extending and including NGC 3447A. The overall velocity field does not emphasise any significant rotation pattern, rather a small velocity gradient between NGC 3447 and NGC 3447A. Our simulation, detached from a large grid explored to best-fit the global properties of the system, suggests that this arises from an encounter between two halos of equal mass.

Conclusions. NGC 3447 and NGC 3447A belong to the same halo, NGC 3447A being a substructure of the same disk including NGC 3447. The halo gravitational instability, enhanced by the encounter, fuels a long-lived instability in this dark-matter-dominated disk, driving the observed morphology. The NGC 3447/NGC 3447A system may warn of a new class of "false pairs" and the potential danger of a misunderstanding of such objects in pair surveys that could produce a severe underestimate of the total mass of a system.
\end{abstract}

Key words. galaxies: groups: individual: LGG 225 - galaxies: individual: NGC 3447/NGC 3447A - galaxies: interactions galaxies: kinematics and dynamics - galaxies: photometry - galaxies: evolution

\section{Introduction}

In the context of group evolution, the Local Group (LG) offers clear examples of ongoing interaction and/or accretion episodes. Both observations and models suggest that the galaxy evolution in the LG is highly dependent on the dynamic properties of the group. Signatures of disruption and accretion of small galaxies are witnessed in the form of gaseous and stellar tidal streams around/onto the accreting galaxy (i.e., the Milky Way, MW hereafter), fossil relics of a mass transfer activity. In recent years several relics have been found in the MW in the form of stellar streams (see e.g. Odenkirchen et al. 2001; Yanny et al. 2003; Belokurov et al. 2006; Grillmar et al. 2006). Concerning M 31(NGC 224 or the Andromeda galaxy), Block et al. (2006) found that, in addition to several well-known signatures of interaction like the warped disk and the ring at about $10 \mathrm{kpc}$, a second

\footnotetext{
$\star$ The reduced images and datacubes are only available at the CDS via anonymous ftp to cdsarc.u-strasbg. fr (130.79.128.5) or via http://cdsarc.u-strasbg.fr/viz-bin/qcat?J/A+A/610/A8
}

ring with a projected dimension of $1.5 \times 1.5 \mathrm{kpc}$ is present. They suggest that both the ring and the warped disk originate from a head-on collision with M 32 (NGC 221) that took place about 210 Myr ago (see e.g. Gordon et al. 2006, and references therein).

In order to place the observed properties of the LG galaxies in the general evolutionary framework of loose groups, we analyse a large sample of galaxy groups spanning a wide range of properties, in richness of member galaxies, velocity dispersion, morphological types and spatial distribution with a multiwavelength approach (Marino et al. 2010, 2013, 2016). In this context, the case of the interacting pair NGC 3447/NGC 3447A, alias VV 252, (Fig. 1) a member of the LGG 225 group (Garcia 1993) in the Leo cloud, is of particular interest for our project (Marino et al. 2010; Mazzei et al. 2014a). This group is at a distance of about $15 \mathrm{Mpc}$ (Marino et al. 2010; Mazzei et al. 2014a). NGC 3447 and NGC 3447A are considered members of a pair composed of a late-barred spiral galaxy, NGC 3447, and an irregular, smaller and fainter companion, 1.2 mag fainter in 
A\&A 610, A8 (2018)

Table 1. Swift-UVOT images: exposure times and integrated magnitudes in the $[\mathrm{AB}]$ system.

\begin{tabular}{cccc}
\hline \hline Filter & $W 2$ & $M 2$ & $W 1$ \\
\hline $\begin{array}{c}\text { Exp. times [s] } \\
\text { mag [AB] }\end{array}$ & $13.95 \pm 0.10$ & $13.93 \pm 0.11$ & $13.83 \pm 0.10$ \\
\hline
\end{tabular}

Notes. Magnitudes corrected for galactic extinction following Roming et al. (2009).

the $B$-band following the NASA/IPAC Extragalactic Database $\left(\mathrm{NED}^{1}\right)$. The morphology details change with the wavelength, highlighting different stellar populations. Both optical and ultraviolet (UV) images show two distinct components, NGC 3447 and NGC $3447 \mathrm{~A}$, connected by a sort of filament. UV bands map out the star forming complexes whose shape, in the outskirts of NGC 3447 at a radius of $\sim 110^{\prime \prime}$, resembles a ring. The global morphology suggests, as a working hypothesis, an ongoing head-on encounter.

Therefore, the study of this nearby system allows us to investigate in great detail the effect of the interactions/collisions on the evolution of the intermediate/low-luminosity galaxies in LG analog (LGA) environments. This work aims to contribute to the on-going studies of galaxy evolution in low-density environments which are favourable habitats to highly effective encounters. The paper is organised as follows. In Sect. 2 we describe observations and reduction of our Fabry-Perot two-dimensional (2D) $\mathrm{H} \alpha$ kinematical data, UV Swift UVOT, and Sloan Digital Sky Survey (SDSS) optical images. Section 3 highlights our photometric and kinematical results which are used to constrain a large grid of smooth particle hydro-dynaminc (SPH) simulations with chemo-photometric implementation (SPH-CPI simulations, hereafter). The simulation which best fits the global properties we derived, that is, the global SED extended over four orders of magnitude in wavelength, the absolute magnitude, the morphology, and the velocity field, provides us with a consistent evolutionary framework of the formation and evolution of the whole system, NGC 3347A/NGC 3447. These points are expanded in Sect. 4. Discussion and conclusions are presented in Sect. 5, and in the Appendix we review and upgrade our knowledge of the environment of the NGC 3347A/NGC 3447 system.

\section{Observations and data reduction}

\subsection{Swift-UVOT UV observations}

UVOT is a $30 \mathrm{~cm}$ telescope on the Swift platform operating both in imaging and spectroscopic modes (Roming et al. 2005). UV images of the NGC 3447/NGC 3447A system come from the Swift archive $^{2}$ in the three available filters, $W 2$ ( $\left.\lambda_{0} 2030\right), M 2$ $\left(\lambda_{0} 2231\right)$, and $W 1\left(\lambda_{0} 2634\right)$. Description of the filters, point spread functions (PSFs) (FWHM 2'.92 for W2, 2'.45 for $M 2$, 2 '.37 for W1), and calibrations are discussed in Breeveld et al. (2010, 2011).

UVOT data obtained in imaging mode with a $2 \times 2$ binning, result in $1 \% 004 /$ pixel, and total exposure times reported in Table 1. We used the photometric zero points provided by Breeveld et al. (2011) to convert UVOT count rates into the AB magnitude system (Oke 1974): $z p_{W 2}=19.11 \pm 0.03, z p_{M 2}=$ $18.54 \pm 0.03, z p_{W 1}=18.95 \pm 0.03$.

UVOT is a photon-counting instrument and is therefore subject to coincidence loss when the throughput is high, whether this is due to background or source counts, which

\footnotetext{
1 http://ned.ipac.caltech.edu

2 www.asdc.asi.it/mmia/index.php?mission=swiftmastr
}

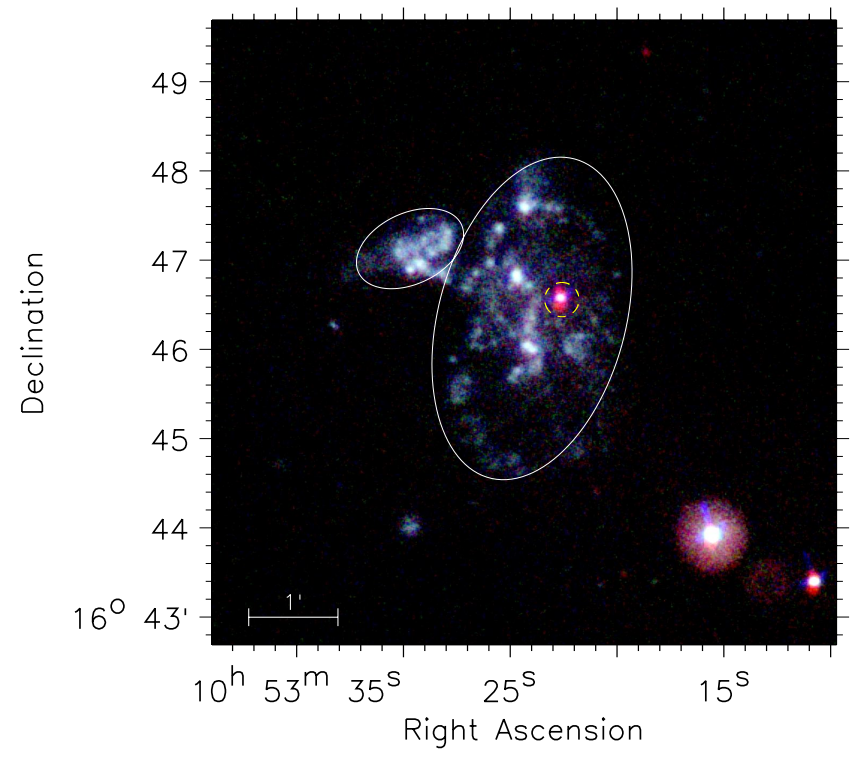

Fig. 1. Colour composite UV image in the Swift UVOT filters, $W 2$ blue, $M 2$ green and $W 1$ red, of the pair system, NGC 3447 and NGC 3447A (white ellipses). The emission within the dashed circle corresponds to the SNa 2012th (Yamanaka et al. 2014), masked in our photometric analysis (Sect. 2.1).

may result in an undercounting of the flux. This effect is a function of brightness of the source and affects the linearity of the detector. Without binning, count rates less than 0.007 counts $\mathrm{s}^{-1}$ pixel $^{-1}$ are affected by at most $1 \%$ and count rate less than 0.1 counts $\mathrm{s}^{-1}$ pixel $^{-1}$ by at most $12 \%$ due to coincidence loss (Breeveld et al. 2011, their Fig. 6). These latter values become 0.028 and 0.4 counts $\mathrm{s}^{-1}$ pixel $^{-1}$ respectively, accounting for $2 \times 2$ binning. We checked for coincidence loss in all our images finding that these are not affected by the problem.

Our images are the sum of several (dithered and rotated) frames. This sum tends to smooth out large-scale inhomogeneities in the final frame. Our target covers a limited portion of the $17^{\prime} \times 17^{\prime}$ of the UVOT field of view (FoV) and the background can be well evaluated around it.

Surface photometry has been performed as described in Rampazzo et al. (2017), that is, using the ELLIPSE (Jedrzejewski 1987) fitting routine in the STSDAS package of Image Reduction and Analysis Facility (IRAF), increasing the size of the apertures logarithmically. Foreground and background objects outside the galaxy body as well as the supernova (SN) on our target (see Fig. 1 ) have been masked. To secure a reliable background estimate, we performed the measurement well beyond the galaxy emission. ELLIPSE provides the semi-major axis lengths $(a)$, the surface brightness $(\mu)$, the ellipticity $(\epsilon)$, the position angle (PA), and the isophotal shape $\left(a_{4} / a\right)$ (Bender et al. 1989; Capaccioli et al. 1990; Governato et al. 1993).

We derived total apparent $\mathrm{AB}$ magnitudes by integrating the surface brightness within elliptical isophotes. Errors were estimated by propagating the statistical errors on the isophotal 
Table 2. SDSS integrated magnitudes in the $[A B]$ system.

\begin{tabular}{cccccc}
\hline \hline Filter & $u$ & $g$ & $r$ & $i$ & $z$ \\
\hline $\operatorname{mag}[\mathrm{AB}]$ & $13.55 \pm 0.16$ & $12.57 \pm 0.05$ & $12.30 \pm 0.05$ & $12.17 \pm 0.08$ & $11.50 \pm 0.23$ \\
$\operatorname{mag}[\mathrm{AB}]^{1}$ & $13.94 \pm 0.13$ & $12.91 \pm 0.05$ & $12.64 \pm 0.06$ & $12.55 \pm 0.08$ & $11.89 \pm 0.14$ \\
\hline
\end{tabular}

Notes. ${ }^{(1)}$ From Table 4 of Marino et al. (2010).

intensity provided by ELLIPSE. Our UV total magnitudes, reported in Table 1, are corrected for galactic extinction following Roming et al. (2009).

\subsection{SDSS data}

We used $u, g, r, i$, and $z$ images mosaicked by Knapen et al. (2014a). These are taken from SDSS DR7 and SDSS-III DR8 science archives. These homogenised, background subtracted and calibrated images cover at least $3 \times D_{25}$, where $D_{25}$ is the galaxy diameter at $25 \mathrm{mag} \operatorname{arcsec}^{-2}$ in each of the SDSS filters. The pixel size is 0 '.396 $\mathrm{px}^{-1}$; the zero points are provided in the keyword magzpt of the header of each released image. The product we used (PGC 0032694) provides a 10'1 FoV. The $\mathrm{AB}$ integrated magnitudes we derived are reported in Table 2 accounting for galactic extinction correction. In the same table we also report the corresponding $\mathrm{AB}$ magnitudes from Table 4 of Marino et al. (2010). All the magnitudes are corrected for galactic extinction from NED.

\subsection{Two-dimensional $\mathrm{H} \alpha$ kinematical observations and data reduction}

Observations $^{3}$ were carried out with the PUMA Fabry-Perot (FP, hereafter) interferometer at the $2.1 \mathrm{~m}$ telescope of the Observatorio Astronómico Nacional at San Pedro Mártir (OAN-SPM) Baja California, México, (PUMA@2.1-mSPM) on March 13, 2013. The PUMA instrument (Rosado et al. 1995) is formed by a Queensgate ET50 etalon in the pupil of a focal reducer with the Marconi2 $2 \mathrm{~K} \times 2 \mathrm{~K}$ CCD detector as receptor. Marconi2 has a physical pixel size of $13 \mu \mathrm{m}$ and a scale on the sky of 0 '.33/px. We performed $4 \times 4$ spatial binning giving a pixel equivalent of 1 1'3 on the sky. Table 3 provides the journal of observations and characteristics of the interferometer we used.

The PUMA data were reduced using the Adhocw software package $^{4}$ and Computeverything ${ }^{5}$ by Daigle et al. (2006). The first step, before the correction phase, is to perform the standard CCD data reduction by applying bias and flat-field corrections to each frame of the data cube. The object acquisition procedure and the data reduction have been described by Amram et al. (1996) and Daigle et al. (2006); see also Sanchez-Cruces et al. (2015) for the specific case of data cubes acquisition directly related to the PUMA instrument.

In addition, a channel to channel transparency correction was done using several zones around the object. A phase map was built by scanning the narrow $\mathrm{H} \alpha 6563 \AA$ line under the same observing conditions and phase corrected cubes were performed using the Adhocw package. Velocities are measured relative to the derived systemic velocity of $1079 \pm 10 \mathrm{~km} \mathrm{~s}^{-1}$ with

\footnotetext{
3 All Fabry-Perot data (cubes and moment maps) are available at http: //cdsweb.u-strasbg. fr

4 Available at

https://cesam.lam.fr/fabryPérot/index/softwares

5 Available at

http://www . astro. umontreal.ca/ odaigle/reduction/
}

Table 3. Fabry-perot observational setup.

\begin{tabular}{lc}
\hline \hline Fabry-Perot Parameters & Values \\
\hline Telescope & SPM 2.1 m \\
Date & 13th March 2013 \\
Seeing & 1'.5 \\
Instrument & PUMA-FP \\
Detector & CCD Marconi \\
Pixel size & $1^{\prime \prime .3} /$ pix \\
Calibration lamp $(\lambda)$ & $6562.78 \AA$ \\
Resolution $(\lambda / \Delta \lambda)$ & 8031 \\
Filter Central Wavelength & $6607 \AA$ \\
Filter Transmission & $66 \% @ 6586 \AA$ \\
Filter FWHM $(\Delta \lambda)$ & $89 \AA$ \\
Finesse@H $\alpha$ & 24 \\
Interferometer order@H $\alpha$ & 330 \\
Free spectral range@ $\mathrm{H} \alpha$ & $19.7 \AA-911 \mathrm{~km} \mathrm{~s}^{-1}$ \\
Number of scanning steps & 47 \\
Sampling step & $0.43 \AA-19.38 \mathrm{~km} \mathrm{~s}^{-1}$ \\
Interferometer FWHM $(\delta \lambda)$ & $0.82 \AA$ \\
Total exposure time $(\mathrm{s})$ & 11280 \\
\hline
\end{tabular}

Notes. ${ }^{(a)}$ Seeing estimated via an unbinned direct image with a pixel scale of $0.33 " / p x$.

an accuracy of $10 \%$. The signal measured along the scanning sequence was separated into two parts: (i) an almost constant level produced by the continuum light in a $89 \AA$ bandpass around $\mathrm{H} \alpha$ (continuum map, not shown but obtained and analysed as a part of the products of the FP datacube) and (ii) a varying part produced by the $\mathrm{H} \alpha$ line ( $\mathrm{H} \alpha$ integrated flux map). The continuum is computed by taking the mean signal outside the emission line. The $\mathrm{H} \alpha$ integrated flux map was obtained by integrating the monochromatic profile in each pixel. Strong OH night-sky lines passing through the filter were subtracted by determining the level of emission away from the galaxies (Laval et al. 1987). In order to improve the signal-to-noise ratio $(\mathrm{S} / \mathrm{N})$ we used both spectral (rectangular, three channels) and a box car $(5 \times 5$ pixels) spatial smoothing in order to minimise the smoothing effects of the spatial resolution. The velocity estimation is done by a barycentric measurement of the velocity from the identified emission profile. The dispersion velocity was estimated by computing the FWHM of the profile, dividing by $2 \times(2 \ln 2)^{1 / 2}$ and correcting from the instrumental and thermal broadening. The instrumental broadening, estimated using the calibration lamp, is $\sigma_{\text {inst }}=18 \mathrm{~km} \mathrm{~s}^{-1}$. We used $\sigma_{\text {th }}=9.1 \mathrm{~km} \mathrm{~s}^{-1}$ from Bordalo et al. (2009) and the previous value of $\sigma_{\text {inst }}$.

\section{The NGC 3447 system: photometric and kinematic properties}

\subsection{Photometric properties}

The UV integrated magnitudes, corrected by galactic extinction are given in Table 1. Figure 2 (left panel) highlights the UV 

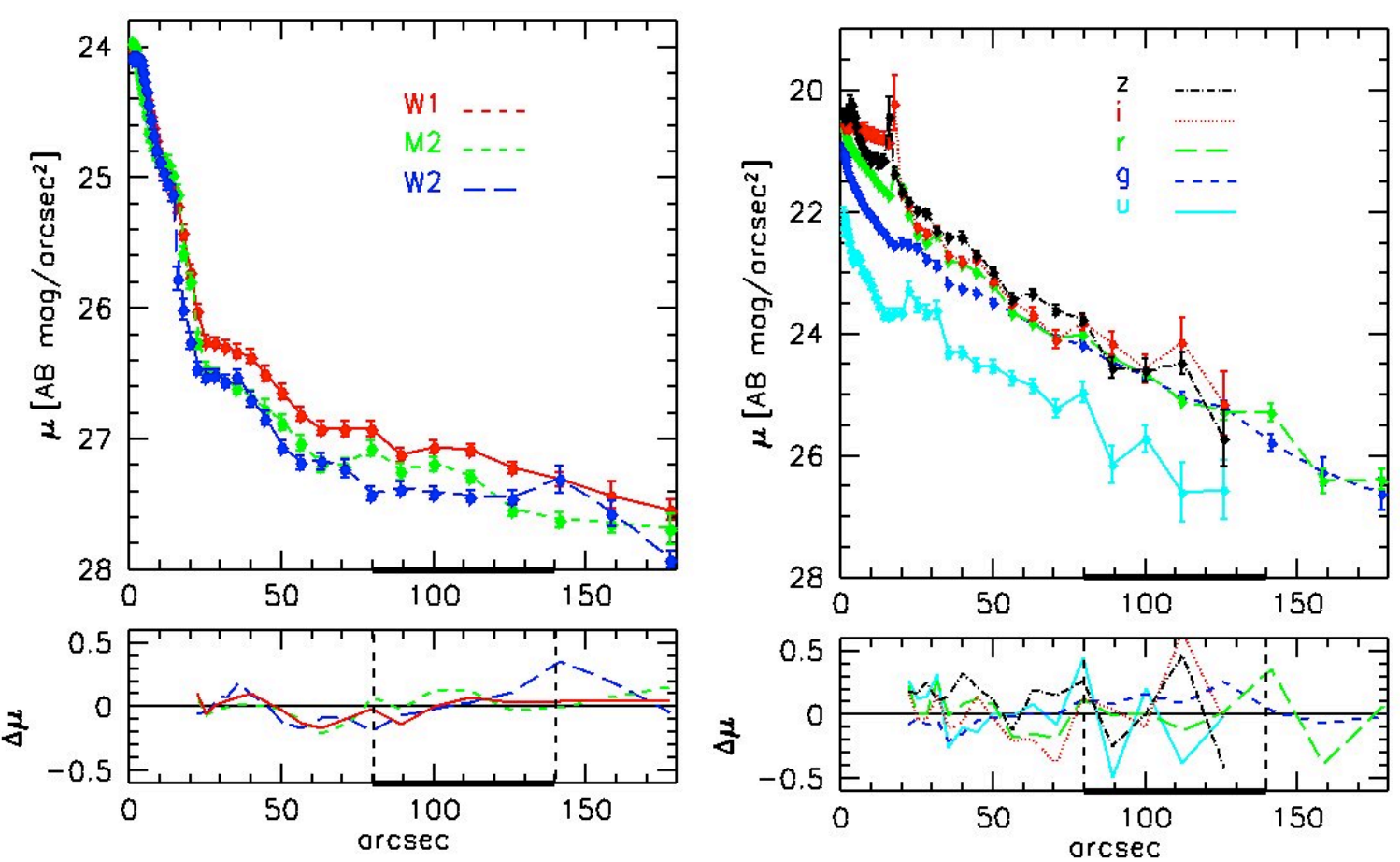

Fig. 2. Surface-brightness profiles in the UVOT $W 2, M 2$ and $W 1$ bands (left) and SDSS, $u, g, r, i$, and $z$ (right). All the profiles include NGC 3447 A, extending from $80^{\prime \prime}$ to $140^{\prime \prime}$, as emphasised (bold) in the figure. The Sérsic fit of all the profiles provides $n=1.4$. The model has been subtracted from the original profile and the residuals are shown in the bottom panels. The spike arising at $18^{\prime \prime}$, from $r$ - to $z$-bands, corresponds to the bright $\mathrm{H} \alpha$ region North of the bar. Profiles are not corrected for galactic extinction and truncated when the error bars exceed 0.5 mag.

luminosity profiles. These profiles have been fitted using an almost exponential disk $(n=1.4)$. The model, given by a Sérsic law, has been subtracted from the original profiles and residuals for all the UVOT filters are shown in the bottom left panel of Fig. 2. In the fit, the bar has been masked as well as the region influenced by the instrumental PSF. For this reason the residuals are shown starting from $20^{\prime \prime}$, where the disk clearly emerges $(n=1.4)^{6}$. Figure 2 (right panel) shows the brightness profiles in the SDSS bands and the residuals by performing the same fit as in the UV bands. We derive $n=1.4$ also in this case. The residuals do not show any significant trend, both in the UV as well as in the SDSS bands, in particular in the region covered by NGC 3447A (emphasised in Fig. 2).

There are no $U, B, V$ magnitudes for this galaxy in the RC3 catalogue. The Hyperleda catalogue reports: $M_{B}=-17.28 \pm$ $1.11 \mathrm{mag}$. The large error includes uncertainties in the observed magnitude (13.90 \pm 0.58 mag corrected for galactic extinction), internal extinction, inclination effect, and distance modulus estimate $( \pm 0.94 \mathrm{mag})$. The AB integrated magnitudes that we derived from optical SDSS images (Table 2) are 0.3 mag brighter, on average, than the total magnitudes of Marino et al. (2010, their Table 4) which, as outlined by the same authors, are estimated in a reduced area because the system is near the edge of their SDSS image.

The distance of NGC 3447 is very uncertain (30\%). From Extragalactic Distance Database (EDD) we derive the largest value, 20.9 Mpc, whereas following our work on LGG 225 (Marino et al. 2010; Mazzei et al. 2014a), we assign to this system a distance of $\simeq 15 \mathrm{Mpc}$. This choice is consistent with the value of $H_{0}=75 \mathrm{~km} \mathrm{~s}^{-1} \mathrm{Mpc}^{-1}$ and the systemic velocity in

\footnotetext{
6 We remind here that the Sersic law is a generalisation of the de Vaucouleurs $r^{1 / 4}$ law (de Vaucouleurs 1948) where the value $n=4$ is typical of Ellipticals and bulges, $n=1$ of exponential disks (Freeman 1970), and $n=0.5$ of a Gaussian profile.
}

Sect. 3, as well as with the other cosmological parameters, $\Omega_{\Lambda}=0.73$, and $\Omega_{\text {bar }}=0.27$ given by the NED catalogue. This implies an uncertainty in its total absolute magnitude of $-0.72 \mathrm{mag}$. Therefore, accounting for this distance, the g magnitude in Table 2, and the Windhorst et al. (1991) filter transformation, $B=g+0.51+0.6 \times(g-r)$ giving $B=13.25 \mathrm{mag}$, we derive the absolute magnitude in the $B$-band: $M_{B}=-17.63 \pm 0.72 \mathrm{mag}$. Unfortunately, a recent study of the SNa 2012ht in NGC 3447 by Yamanaka et al. (2014), which classified this SN as a transitional SN Ia between normal and subluminous SNae Ia, does not provide any distance (modulus) estimate.

\subsection{Two-dimensional $\mathrm{H} \alpha$ kinematic analysis: moment maps}

In the top-left panel of Fig. 3, we show the SDSS $r$-band image of the same field observed with FP. The velocity map of NGC 3447 (top-right panel) does not show any regular disk rotation pattern, rather a small velocity gradient, between $1043 \mathrm{~km} \mathrm{~s}^{-1}$ in the southern tip and $1085 \mathrm{~km} \mathrm{~s}^{-1}$ in the North. NGC 3447A also shows a velocity gradient between $1090 \mathrm{~km} \mathrm{~s}^{-1}$ in the South East and $1130 \mathrm{~km} \mathrm{~s}^{-1}$ in the North West.

The SDSS $r$-band centre of NGC 3447 does not correspond to any emitting region in the $\mathrm{H} \alpha$ monochromatic map (Fig. 3 left, up and bottom panels). The same map shows three very bright emitting regions, one South and one North of the optical central bar, and the third to the South East region of NGC 3447A.

The velocity dispersion map (bottom-right panel) shows that almost all the emitting regions have supersonic velocity dispersion, between $20 \mathrm{~km} \mathrm{~s}^{-1}$ and $45 \mathrm{~km} \mathrm{~s}^{-1}$. We estimated the mean velocity dispersion of the all objects is $30 \pm 6 \mathrm{~km} \mathrm{~s}^{-1}$, outlining the highly supersonic nature of the ionised gas in this galaxy. By comparing the monochromatic emission and velocity dispersion maps, we note a different behaviour for each of the three brightest regions of the monochromatic map. The one in the South shows the lower velocity dispersion with respect 

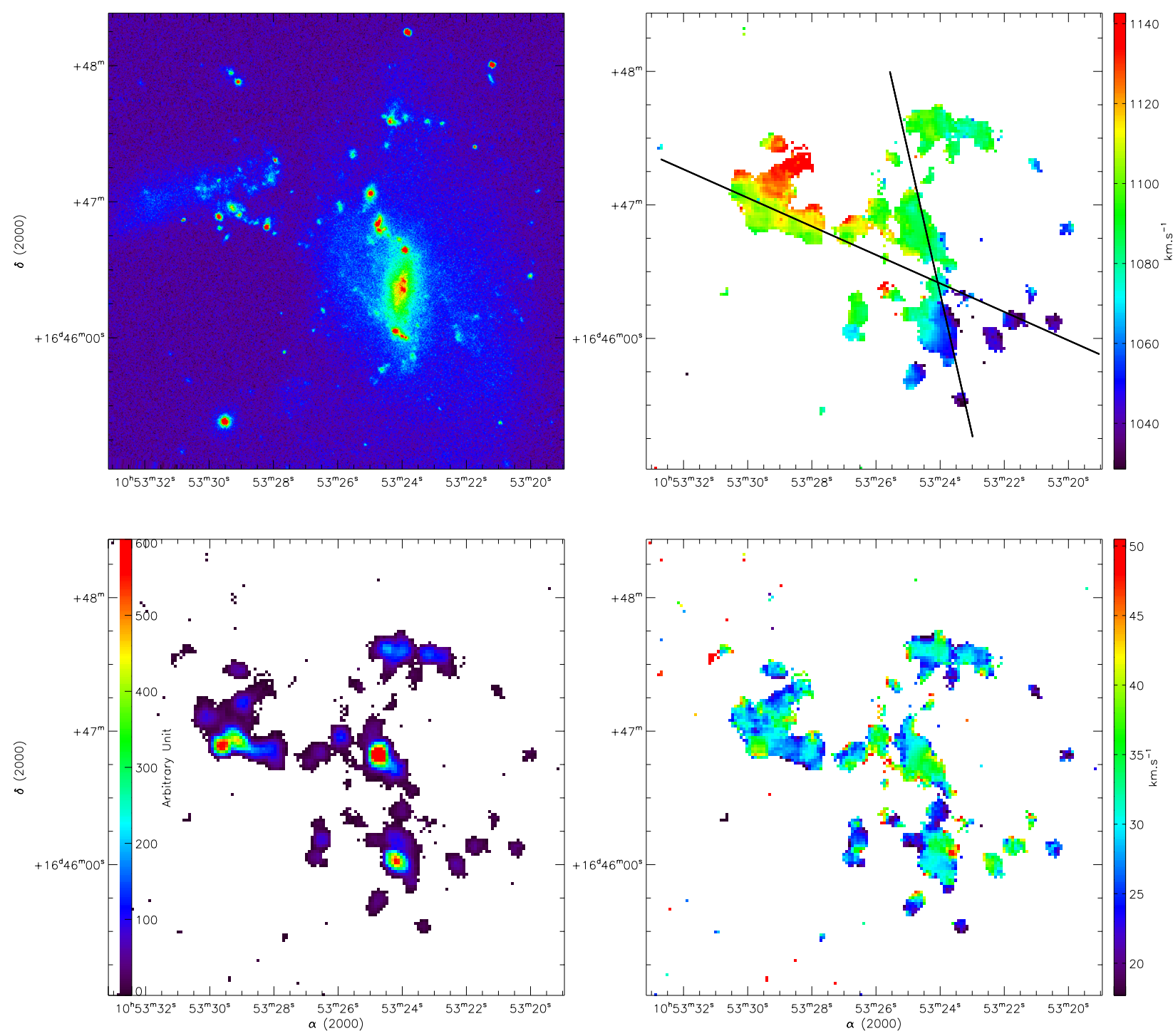

Fig. 3. Top: SDSS $r$-band image of NGC 3447/3447A (left) and the velocity field of $\mathrm{H} \alpha$ emission (right). Bottom: the H $\alpha$, monochromatic emission map (left) and its velocity dispersion map (right). All panels correspond to the same 3.5 $\times 3.5$ field, and are on the same spatial scale with North on the top and East to the left. Black solid lines in the top right panel show the position angles along which the velocity profiles, shown in Fig. 4, are derived.

to its surrounding, the second, in the East, shows the opposite trend, and the third, in the centre, does not show any clear pattern. The behaviour of the southern region is the same as that found in several studies of giant H II regions and H II galaxies (Muñoz-Tuñón et al. 1996; Bordalo et al. 2009) and in interacting pairs (Plana et al. 2017). No definite answer has been firmly established for this feature, possibly due to the presence of expanding shells (Tenorio-Tagle et al. 1993). We performed a check of several profiles in order to find out if multiple components could be present. We found that the profiles are fairly symmetric with no obvious multiple components. This could come from the superposition in the line of sight of different H II regions/clouds. Due to our insufficient spectral (due to the spectral smoothing in order to gain $\mathrm{S} / \mathrm{N}$ ) and spatial resolution, no further conclusions can be drawn.

For the purpose of constraining our simulation, we obtained the radial velocity position profiles, shown in Fig. 4, along the lines of sight highlighted in Fig. 3 (top right panel), corresponding to (i) the north eastwards major axis PA, $\mathrm{PA}=14.3^{\circ}$
(Hyperleda catalogue), and (ii) $\mathrm{PA}=65^{\circ}$, the line of sight connecting the centre of NGC 3447, selected from the $r$-band SDSS image, to NGC 3447A, both accounting for a semi-angle sector of $25^{\circ}$ (Amram et al. 2007, their Fig. 9c). The velocities are estimated in different crowns of two pixels each.

To summarise, we do not find any kinematic signature proving that NGC 3447 and NGC 3447A have different velocity fields. The velocity field does not show any rotation pattern. The velocity gradient across the entire system is too weak and irregular to be considered as a rotation pattern, although the South West region appears slightly approaching and the North East slightly receding. Our results point towards the idea that patchy and distorted features are different regions of a single system.

\section{Comparison with simulations}

We explored the evolutionary scenario of the NGC 3447/ NGC 3447A system exploiting a large set of SPH-CPI simulations of galaxy encounters and/or mergers, including a 

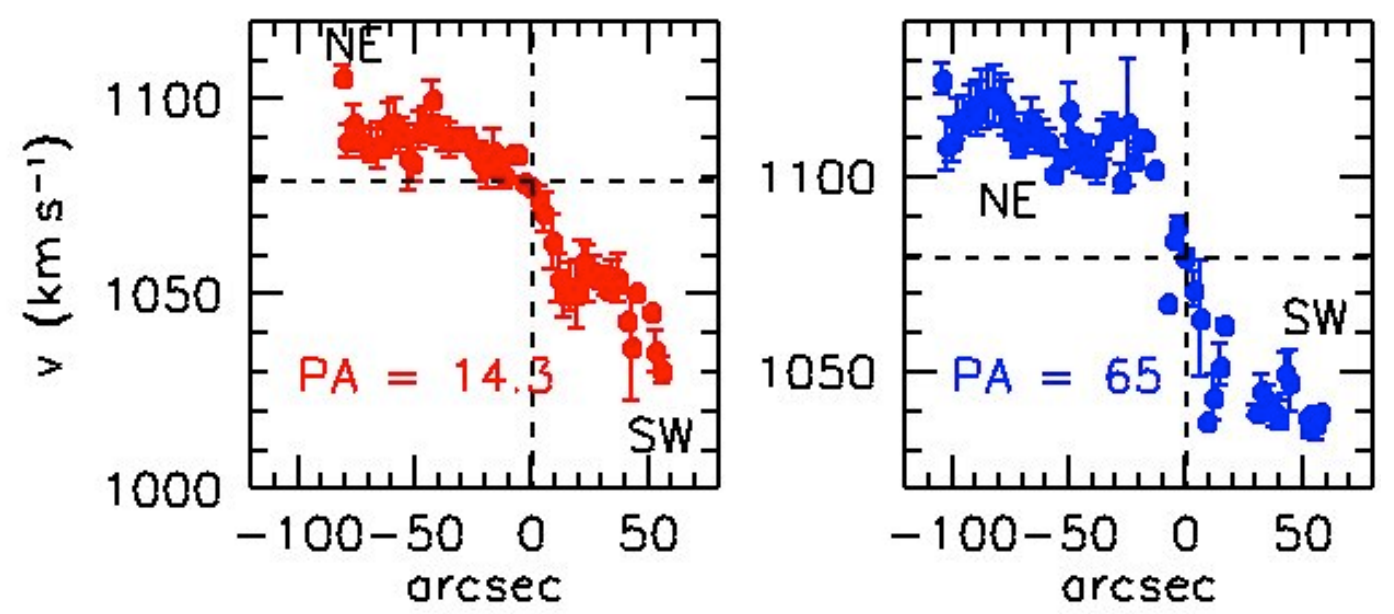

Fig. 4. Velocity profiles along the lines of sight in Fig. 3 (top left panel) corresponding to $\mathrm{PA}=14.3^{\circ}$, and $\mathrm{PA}=65^{\circ}$ which connects NGC $3447 \mathrm{~A}$; horizontal dashed lines indicate the derived systemic velocity $\left(1079 \mathrm{~km} \mathrm{~s}^{-1}\right.$, Sect. 2.3).

chemo-photometric code based on evolutionary population synthesis (EPS) models. The general prescriptions of our SPH-CPI simulations are reported in several previous papers (Mazzei et al. 2014a,b; Buson et al. 2015, and references therein). All the simulations start from collapsing triaxial systems composed of dark matter (DM) and gas in different proportion and different total masses, as in Mazzei \& Curir (2003, MC03 hereafter). The simulated halos have all the same initial conditions, that is, virial ratio $(0.1)$, average density, and spin parameter. In more detail, each system is built up with a spin parameter, $\lambda$, given by $\mid \boldsymbol{J} \| E^{0.5} /\left(G M^{0.5}\right)$, where $E$ is the total energy, $\boldsymbol{J}$ is the total angular momentum, and $G$ is the gravitational constant; $\lambda$ is equal to 0.06 and aligned with the shorter principal axis of the DM halo. The initial triaxiality ratio of the DM halos, $\tau=\left(a^{2}-b^{2}\right) /\left(a^{2}-c^{2}\right)$, is 0.84 (Mazzei et al. 2014a,b) where $a>b>c$ (Warren et al. 1992). This $\tau$ value is different from the fiducial value of $\tau=0.58$ adopted by MC03 and motivated to be closer to the initial condition of cosmological halos as in Warren et al. (1992), Curir et al. (2006, their Table 1), and Schneider et al. (2012).

The simulations include self-gravity of gas, stars and DM, radiative cooling, hydrodynamical pressure, shock heating, viscosity, star formation, feedback from evolving stars and type-II SNae, and chemical enrichment.

The initial mass function (IMF) is of Salpeter type (Salpeter $1955)$ with upper and lower mass limit 100 and $0.01 M_{\odot}$ respectively, as in Curir \& Mazzei (1999a) and MC03.

All the model parameters had been tuned in previous papers devoted to analysing the evolution of isolated collapsing triaxial halos, initially composed of DM and gas (Curir \& Mazzei 1999a; Mazzei 2003, and MC03). In those papers, the role of the initial spin of the halos, their total mass and gas fraction, triaxiality ratio, as well as different IMFs, particle resolutions, SF efficiencies, and values of the feedback parameter, were all examined. The integrated properties of simulated galaxies, stopped at $15 \mathrm{Gyr}$, that is, their colours, absolute magnitudes, metallicities, and mass to luminosity ratios, have been successfully compared with those of local galaxies (Curir \& Mazzei 1999a, e.g. their Fig. 17; Mazzei 2003, 2004, their Fig. 8). In particular, a slightly higher star formation rate (SFR) compared with the other possibilities examined, arises from our IMF choice (see MC03 their Fig. 1); this allows for the lowest feedback strength (63\% less than that in the same simulation with the lower mass limit $0.1 M_{\odot}$ ), and for the expected rotational support when disk galaxies are formed (MC03).

Each simulation self-consistently provides morphological, dynamic, and photometric evolution, that is, the spectral energy distribution (SED), at each evolutionary time, that is, at each snapshot. The time step between individual snapshots is 0.037 Gyr . The SED that we derive accounts for chemical evolution, internal extinction, and re-emission by dust in a selfconsistent way. This extends at least over four orders of magnitude in wavelength, that is, from 0.06 to $1000 \mu \mathrm{m}$.

We point out that the resulting SFR, the driver of the evolution of the global properties of the simulated galaxies, converges when the initial particle number is about $10^{4}$ (Fig. 1 of Mazzei \& Curir 2003; Christensen et al. 2010, 2012).

We performed a grid of simulations of mergers and encounters starting from systems built up with the same initial conditions and using the same parameters tuned in the above cited papers, as described in Mazzei et al. (2014a,b). The set performed accounts for different masses (from $10^{13} M_{\odot}$ to $10^{10} M_{\odot}$ for each system), mass ratios (from 1:1 to 10:1), gas fraction (from 0.1 to 0.01 ), and particle number (initial number of gas and DM particles from 40000 to 220000 ). By seeking to exploit a wide range of orbital parameters, we varied the orbital initial conditions in order to have, for the ideal Keplerian orbit of two points of given masses, the first peri-centre separation, $p$, equal to the initial length of the major axis of the more massive triaxial halo down to $1 / 10$ of the same axis. For each peri-centre separation we changed the eccentricity in order to have hyperbolic orbits of different energy. The spins of the systems are generally parallel with one another and perpendicular to the orbital $(X Y)$ plane, so we studied direct encounters. Cases with misaligned spins have also been analysed in order to investigate the effects of the system initial rotation on the results.

From our grid of SPH-CPI simulations we single out the simulation that simultaneously (i.e. at the same snapshot) accounts for the following observational constrains which correspond to the global properties of NGC 3447A/NGC 3447: i) total absolute $B$-band magnitude within the range allowed by observations (Sect. 3.1); ii) integrated SED as the observed one; iii) morphology like the observed one in the same bands and with the same spatial scale; iv) velocity field like that derived (Sect. 3.2). The results we present are predictions of the simulation which best reproduces all the previous observational constraints at the same snapshot. This snapshot sets the age of the galaxy. 
Table 4. Input parameters of SPH-CPI simulation of NGC 3447/NGC 3447A.

\begin{tabular}{lllcccccc}
\hline \hline$N_{\text {part }}$ & $\begin{array}{l}a \\
{[\mathrm{kpc}]}\end{array}$ & $p / a$ & $\begin{array}{c}r_{1} \\
{[\mathrm{kpc}]}\end{array}$ & $\begin{array}{c}r_{2} \\
{[\mathrm{kpc}]}\end{array}$ & $\begin{array}{c}v_{1} \\
{\left[\mathrm{~km} \mathrm{~s}^{-1}\right]}\end{array}$ & $\begin{array}{c}v_{2} \\
{\left[\mathrm{~km} \mathrm{~s}^{-1}\right]}\end{array}$ & $\begin{array}{c}M_{\mathrm{T}} \\
{\left[10^{10} M_{\odot}\right]}\end{array}$ & $f_{\text {gas }}$ \\
\hline 80000 & 376 & $1 / 3$ & 505 & 505 & 18 & 18 & 20 & 0.1 \\
\hline
\end{tabular}

Notes. Columns are as follows: (1) total number of initial $(t=0)$ particles, $N_{\text {gas }}=N_{\text {DM }}=N_{\text {part }} / 2$; (2) length of the semi-major axis of the halo; (3) peri-centric separation of the halos in units of the semi-major axis; (4) and (5) distances of the halo centres of mass from the centre of mass of the total system, (6) and (7) velocity moduli of the halo centres in the same frame; (8) total mass of the simulation; (9) initial gas fraction of each halo.
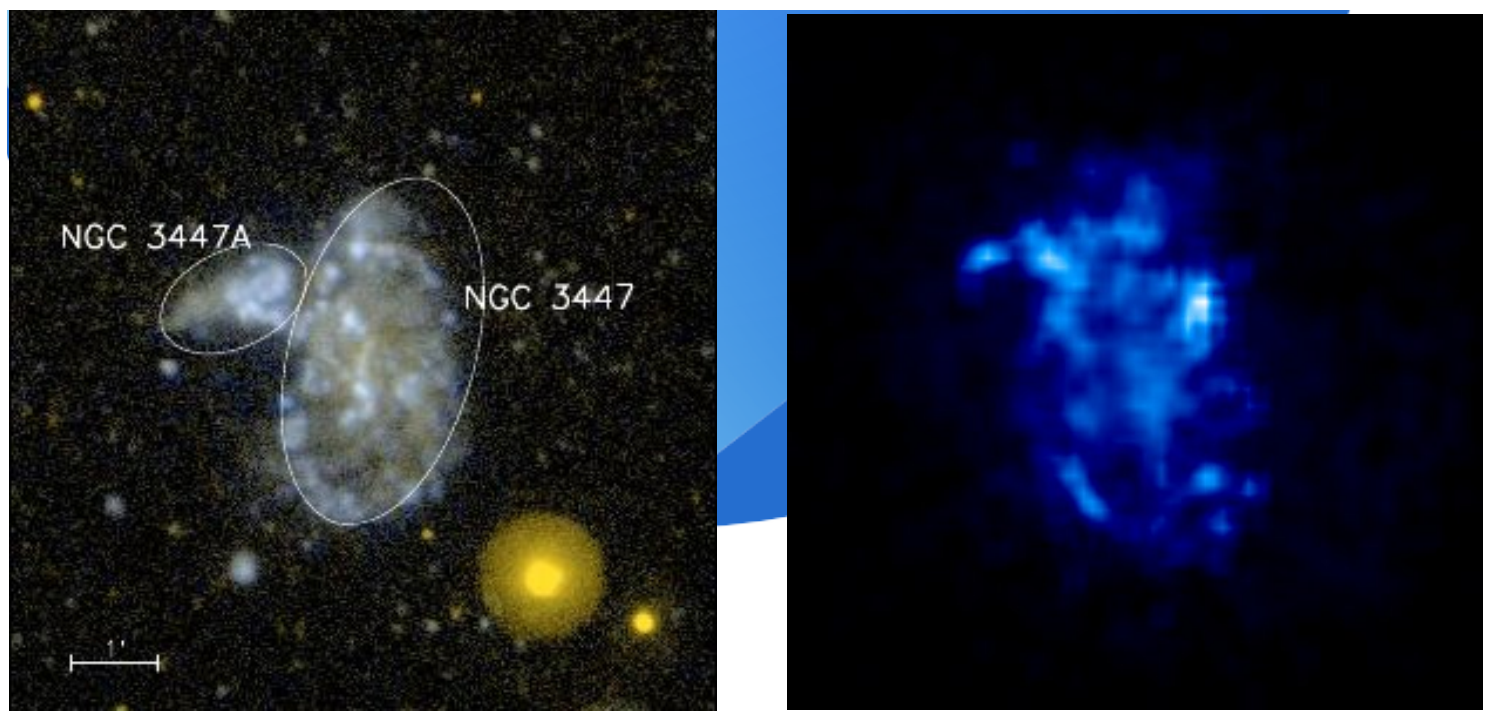

Fig. 5. Comparison between the GALEX NUV (yellow), FUV (blue) composite image, $7^{\prime} \times 7^{\prime}$, from Marino et al. (2010, left panel), and the FUV band luminosity density map ( $X Y$ projection) from the snapshot best fitting the global properties of NGC 3447/NGC 3447A (right panel) normalised to the total flux in the box; maps are on the same spatial scale, and resolution $\left(5^{\prime \prime}\right)$.
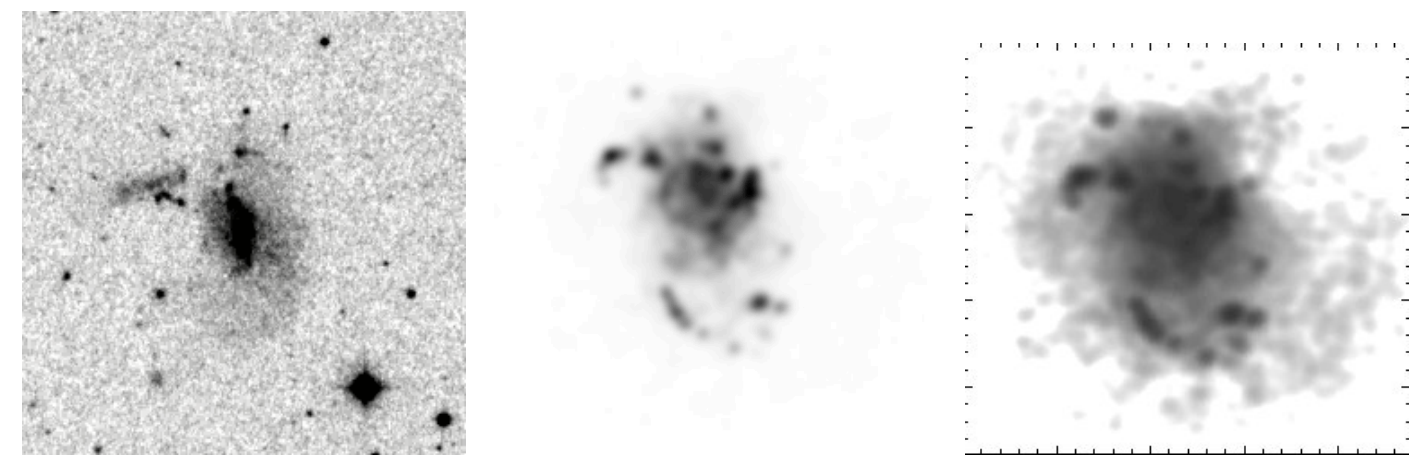

Fig. 6. Comparison between the $B$-band DSS image from NED, $7^{\prime} \times 7^{\prime}$, left panel, and the $B$-band luminosity density maps (XY projection) from the snapshot best fitting the global properties of NGC 3447A/NGC 3447 normalised to the total flux in the box (middle and right panels); maps are on the same spatial scale, and resolution (1'!7), and with different density contrast, 200 (middle) and 1500 (right).

We address the reader to the papers by Mazzei et al. (2014a) where our approach has been applied to early-type galaxies of two groups, USGC 367 and LGG 225, and Mazzei et al. (2014b) where our approach has been exploited to match not only photometric, but also structural (e.g. disk vs. bulge) and kinematical (gas vs. stars) properties of two S0 galaxies, namely NGC 3626 and NGC 1533, to predict their evolution (Spavone et al. 2009; Trinchieri et al. 2012; Bettoni et al. 2012; Buson et al. 2015; Plana et al. 2017).

The initial conditions, that is, particle number, total mass, and gas fraction, together with orbital parameters, of the selected simulation which corresponds to an encounter, are given in Table 4. Moreover, the initial spins of the halos are perpendicular; the initial gas mass resolution is $5 \times 10^{5} M_{\odot}$, that of DM particles is nine times larger, and that of the star forming clusters is $2 \times 10^{4} M_{\odot}$. The gravitational softening is $1,0.5$, and $0.05 \mathrm{kpc}$ for DM, gas, and star particles respectively. The final number of particles at least doubles the initial number in Table 4 .

\subsection{Matching the NGC 3447A/NGC 3447 system properties}

We find that the simulation which best fits the global properties of the whole system, NGC $3447 / \mathrm{NGC} 3447 \mathrm{~A}$, that is, its total $B$-band absolute magnitude, integrated SED (Fig. 7, left panel), morphology (Figs. 5 and 6), and dynamical properties (Fig. 7, middle and right panels), corresponds to an encounter, not to 

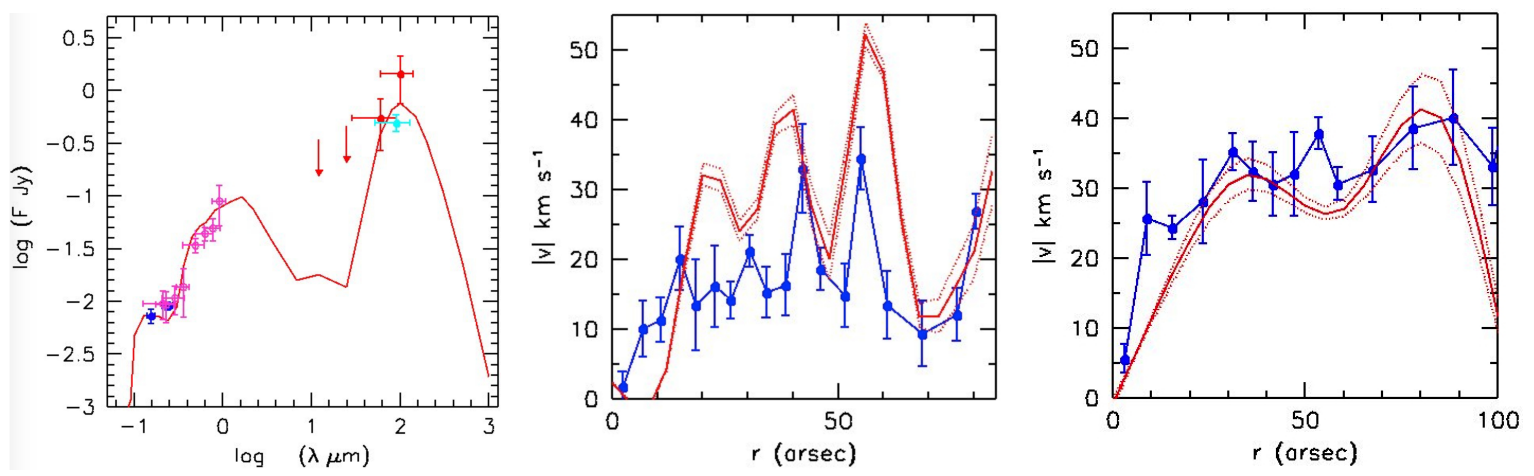

Fig. 7. Left: measured (filled dots) and predicted (red solid line) SED of the NGC 3447A/NGC 3447 system; blue, magenta, red, and azure dots indicate far-UV GALEX, from Marino et al. (2010), Swift-UVOT and SDSS (Tables 1 and 2), IRAS and AKARI-F (Catalog Archive Server, Yamauchi et al. 2011) data, respectively. Middle and right: comparison between the observed velocity profiles along the directions in Fig. 4, PA = $14.3^{\circ}$ (middle) and PA $=65^{\circ}$ (right), with those derived from the selected snapshot in the corresponding directions and within the same semi-angle sector (i.e. $25^{\circ}$ ). The velocity profiles are folded and rebinned within $5^{\prime \prime}$ (blue filled dots) to be compared with the same slices of the simulation (red solid and dotted lines), with the same binning.

a merger, between two halos of equal mass, each of $10^{11} M_{\odot}$ and therefore to a total mass of $2 \times 10^{11} M_{\odot}$ (Table 4). The snapshot which best-fits all the global properties of NGC 3447/ NGC 3447A gives $M_{B}=-17.25 \mathrm{mag}$ and a galaxy age of 12 Gyr. However, the average age of the stellar populations is younger, increasing from 3 to $7 \mathrm{Gyr}$ as increasing the radius up to $15 \mathrm{kpc}$. Furthermore, the age of stellar populations weighted by $B$-band luminosity is $1.3 \mathrm{Gyr}$, and $0.9 \mathrm{Gyr}$ weighted by $U$ band luminosity, both are almost constant within the galaxy. The mass of stars in the same region as in Figs. 5 and 6, comparing UV and optical morphologies, is $2.2 \times 10^{9} M_{\odot}$; that of the DM 13.7 times larger. Maps are on the same scale as observations, where $7^{\prime} \times 7^{\prime}$ corresponds to about a projected box $(X Y$ projection in this case) of $40 \mathrm{kpc} \times 40 \mathrm{kpc}$ on the stellar mass centre, accounting for a scale of $5.5 \mathrm{kpc} /{ }^{\prime}$ as given by NED for $H_{0}=75 \mathrm{~km} \mathrm{~s}^{-1} \mathrm{Mpc}^{-1}$ (Marino et al. 2010) and the cosmological parameters in Sect. 3.1.

The simulation provides that the companion galaxy, at the time of analysis (i.e. $12 \mathrm{Gyr}$ ), lies about $300 \mathrm{kpc}$ from our target, corresponding to about one degree on the sky accounting for the previous scale. Moreover, this is expected to be 0.5 mag fainter in the $B$-band.

The far-IR (FIR) SED in Fig. 7 (left panel) accounts for a diffuse dust emission spectrum composed of warm and cold dust, both including polycyclic aromatic molecules, as described by Mazzei et al. (1992, 1994). Warm dust is located in regions of high density of radiation field, that is, in the neighbourhood of OB stars (H II regions), whereas cold dust is heated by the interstellar diffuse radiation field. The distribution of the cold dust emission is the same as in the MW (Mazzei et al. 1992), that is, provides a disk of gas and stars, in agreement with our findings in Sect. 3.1. However, the intensity required is 6-7 times larger than the average in our own galaxy, and the warm/cold energy ratio is 0.1 instead of 0.3 . A more detailed FIR coverage is needed to derive strong conclusions concerning these points, however the greater intensity of the diffuse radiation field that we derive, agrees with the low metallicity of stars in this system compared with that in our galaxy since low-metallicity stars are more luminous than stars with solar composition (see for a review Chiosi 2007). The average stellar metallicity provided by the best-fit snapshot is 0.0035 ; almost 5-6 times lower than that in the solar neighbour.

Figure 7 , middle and right panels, compares the observed velocity profiles along the lines of sight in Fig. 4, both folded with respect to the galaxy centre and binned within $5^{\prime \prime}$, with our predictions in the same slices and with the same binning. We folded the velocity profiles although, as we remark in Sect. 3.2, we did not find any rotation curve, rather a velocity gradient that is matched, within errors, by the selected snapshot of our simulation.

Figure 8 compares UV and optical $B$-band projected morphologies of the best-fit snapshot with those one snapshot before $(0.037 \mathrm{Gyr})$ and one after, showing that both NGC 3447A and NGC 3447 are pieces belonging to the same system. NGC 3447A and NGC 3447 belong to a single, distorted and broken disk galaxy. This galaxy corresponds the baryonic component of one halo of our simulation; the second halo, containing a galaxy 0.5 mag fainter $(B$-band) than this, is about 1 degree away from our luminous target. Therefore, such a galaxy cannot appear in any of the previous pictures whose FoV is $7^{\prime} \times 7^{\prime}$.

Figure 9 compares the SEDs and velocity profiles of the same snapshots shown in Fig. 8, all computed with the same recipes as in Fig. 7.

To summarise, the SPH-CPI simulation that we present suggests that NGC 3447 and NGC 3447A are a single system, the result on the baryonic disk of its halo instability enforced by the encounter with a companion halo of equal mass. Following this view, pairs like NGC 3447 and NGC 3447A may be considered to belong to the class of the so-called "false pairs". These latter objects are known to lurk within galaxy associations: the pair members have similar redshifts but they are gravitationally bound to the group, not each other. In this context, our system points towards a possible sub-class of false pairs: although the projected shape of the NGC 3447/3447A system suggests it is a pair, according to our simulation it is a single object. Its patchy structure is connected to the disk gravitational instability triggered by the interaction. In loose groups, interactions are very effective and strongly perturb the galaxy evolution. This is just the environment where the NGC 3447A/NGC 3447 lies. We will deepen the analysis of the environment of this system in Appendix A.

\subsection{Insights into the evolution of the NGC $3447 A / N G C 3447$ system}

Figure 10 (left panel) shows the gas accretion history until the time corresponding to the predicted age of the galaxy, that is 12 Gyr (see section above). This figure refers to a spherical 

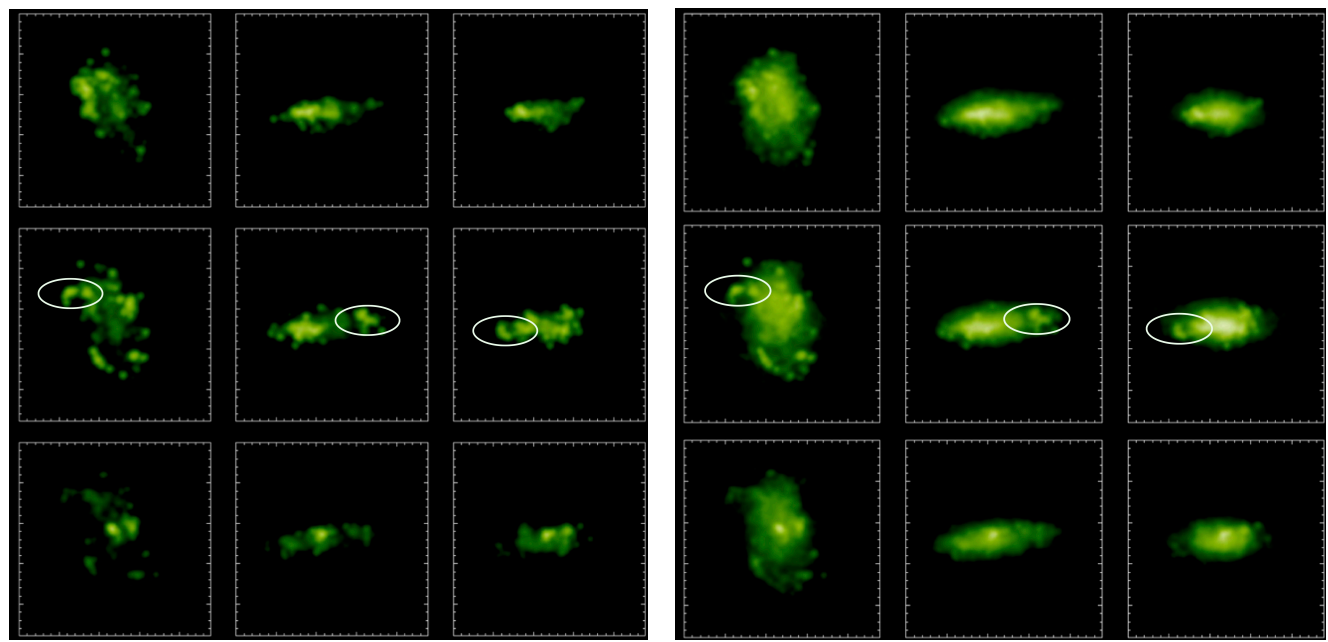

Fig. 8. Projected luminosity density maps, $x y, y z$, and $x z$ from left to right, with the same FoV and resolution as in Fig. 6 . The best-fit snapshot (middle) highlights the region corresponding to NGC 3447A (white ellipse), the snapshot $37 \times 10^{6} \mathrm{yr}$ after is at top, and that $37 \times 10^{6} \mathrm{yr}$ before down. Left: the projected maps in the M2 Swift filter and right, in the $V$-band, each normalised to the total flux inside each box.
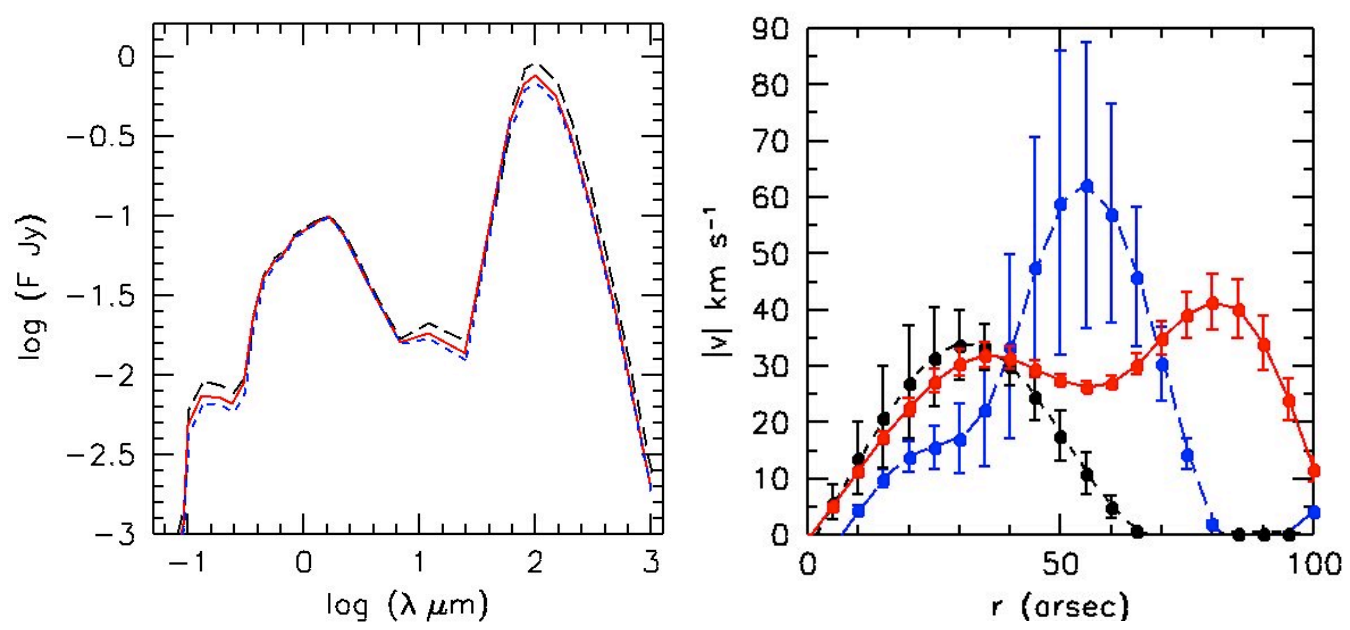

Fig. 9. Comparison of the SEDs (left), and the velocity profiles (right) along PA $=65^{\circ}$, of the best-fit snapshot, (red) solid line, with those of one snapshot $(0.037 \mathrm{Gyr})$ before, (blue) short-dashed line, and one after, (black) long-dashed line.
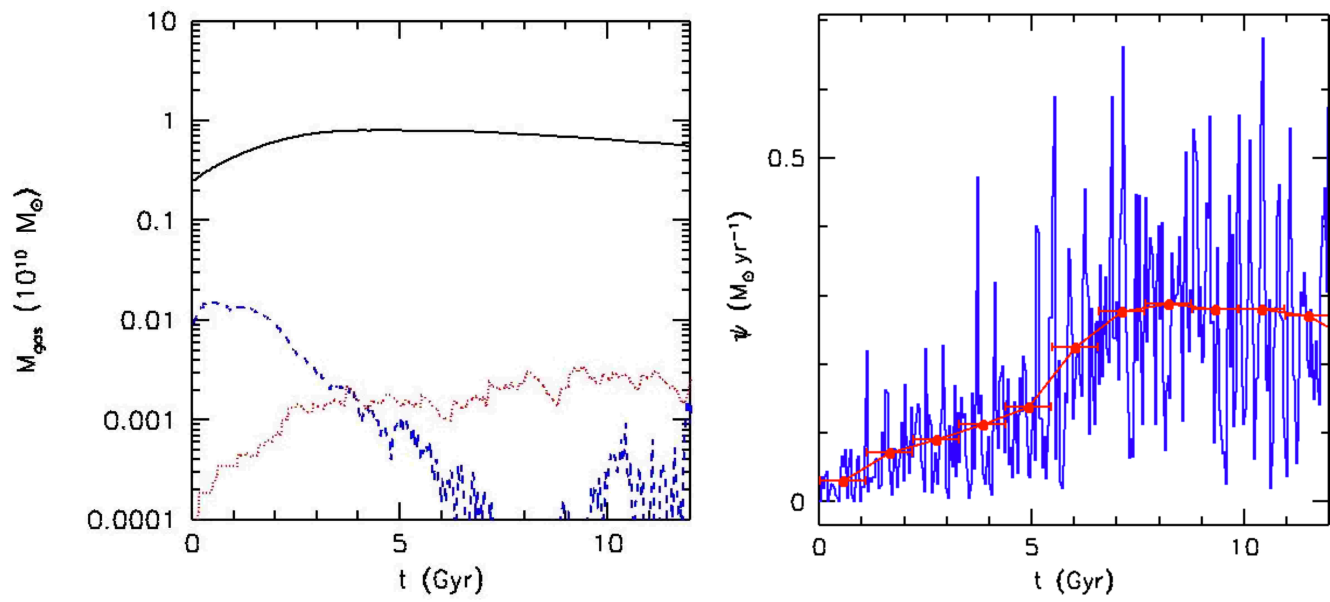

Fig. 10. Evolution of different system properties computed inside a spherical radius of $50 \mathrm{kpc}$ on the $B$-band luminous centre of the simulation matching the global properties of the NGC 3447A/NGC 3447 system. Both panels stop at the actual age of the galaxy, that is, 12 Gyr (Sect. 4). Left: the gas accretion history; the (blue) dashed and (red) dotted lines correspond to the gas with temperature $\leq 10^{4} \mathrm{~K}$ and $>10^{6} \mathrm{~K}$ respectively. Right: the SFR (blue solid line) and its behaviour averaging within 1 Gyr (red bins). 

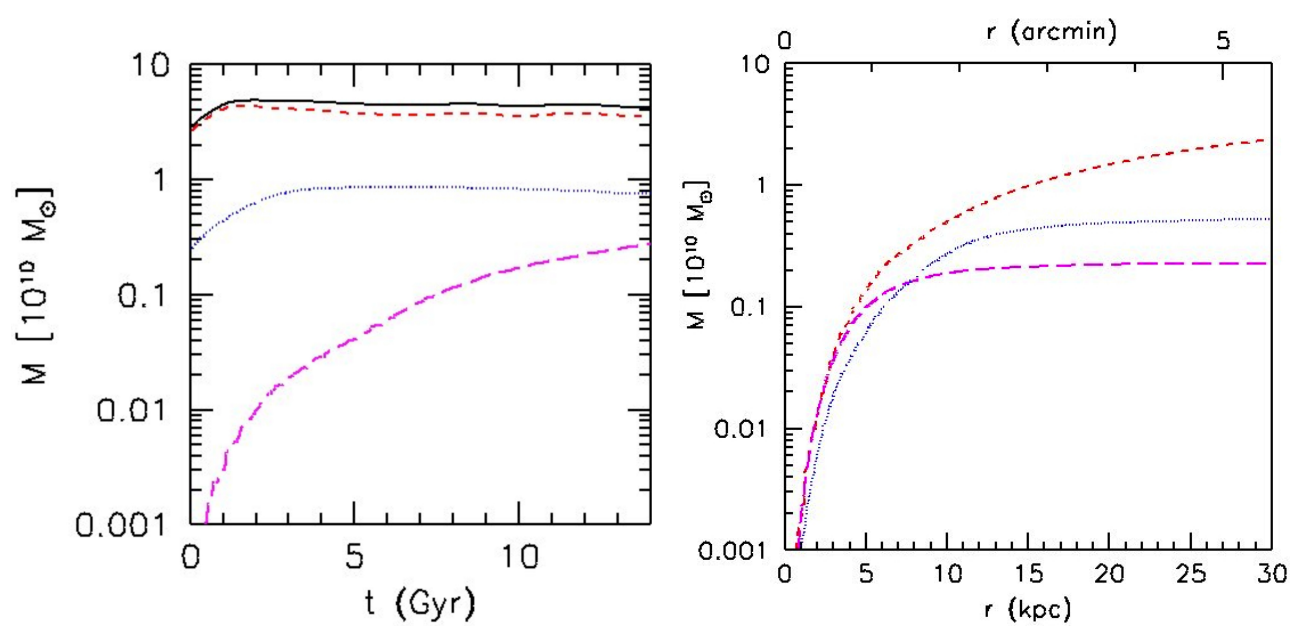

Fig. 11. Left: evolution of the different mass components within the same spherical radius of $50 \mathrm{kpc}$ as in Fig. 10: (black) solid line shows the total mass, (red) short-dashed line the DM, (blue) dotted line the gas, and (magenta) long-dashed line the stellar mass. Right: the mass distribution inside a spherical radius of $30 \mathrm{kpc}$ on the $B$-band luminous centre, at the best-fit age: (red) dashed line for the DM, (magenta) long-dashed for the stars, and (blue) dotted line for the gas component.

radius of $50 \mathrm{kpc}$ on the $B$-band luminous centre of the galaxy. The mass of gas involved in the collapse within this stellar radius is lower than the total gas mass available in the initial volume $\left(1 \times 10^{10} M_{\odot}\right)$. Despite the large gas reservoir, the SFR within the same stellar radius is always below $1 \mathrm{M}_{\odot} / \mathrm{yr}$ (Fig. 10, right panel).

The total SFR predicted by the simulation at the best-fit age, $0.28 M_{\odot} \mathrm{yr}^{-1}$, is in good agreement with our estimates from the FUV luminosity following the recipes of Kennicutt (1998), that is $0.24 M_{\odot} \mathrm{yr}^{-1}$ accounting for the same IMF lower mass limit. Pacifi et al. (2013) found that low-mass galaxies have, on average, a rising star formation history, in good agreement with our prediction. Moreover, low-mass galaxies are susceptible to episodic (bursty) star formation events (Bauer et al. 20013), well reproduced by our simulation.

As discussed in several previous papers (Mazzei \& Curir 2001; Curir et al. 2008, and references therein), in DMdominated disks, as in this case (Fig. 11), the patchy and lopsided morphology of the system, showing a faint inner bar and an outer semi-ring, arises from the disk instability induced by the halo gravitational instability itself, which is enhanced by the interaction. In other words, unrelaxed and/or perturbed halos allow long lasting instabilities in DM-dominated disks (Mazzei \& Curir 2001). Moreover, the barred disk configuration is made fainter by increasing the initial triaxiality ratio of the halo (Mazzei \& Curir 2001; here 0.84, see Sect. 4), in agreement with the photometric properties of this system.

\section{Discussion and conclusions}

This paper investigates the evolution of the NGC 3447A/ NGC 3447 system in the LGG 225 group. This group is a LGA, that is, a group dominated by Spirals and/or a blue galaxy population (Marino et al. 2010; Mazzei et al. 2014a). The morphology of the NGC 3447 A/NGC 3447 system is highly asymmetric, showing a ring-like structure in the outskirts, brighter in the Swift UV bands. Its inner morphology shows a bar, stronger in the optical bands.

We obtained multi-wavelength Swift-UV and SDSS surface photometry, and the $\mathrm{H} \alpha$ velocity field of the NGC 3447A/ NGC 3447 system by performing FP observations via
PUMA@2.1-mSPM. All these data are used to constrain our grid of SPH-CPI simulations of mergers and encounters, to select the simulation which best fits the global properties of NGC 3447A/NGC 3447. All the other model parameters have been tuned in previous papers (Sect. 4).

We find the following results:

The fit of the surface-brightness profiles from far-UV to $i$ band, using a single Sérsic law starting from $20^{\prime \prime}$, that is, outside the PSF effects and bar prevalence, suggests a disk $(n=1.4)$ extended up to including NGC 3447A.

The $\mathrm{H} \alpha$ velocity field of total system does not show any regular velocity rotation pattern. We selected two suitable lines of sight to derive the radial velocity position profiles finding no rotation along the major axis, and almost regular rotation along the line connecting NGC 3447A and NGC 3447.

The integrated $B$-band absolute magnitude, the SED extended from far-UV to far-IR, the morphology, and the 2D kinematics of the whole system are all best fitted by a simulation corresponding to an on-going encounter. Two halos of initial equal masses, each of $10^{11} M_{\odot}$, are still approaching. The interaction modifies the halo potentials so that the evolution of each system differs from the same in the isolated case (Mazzei \& Curir 2003), and induces the galaxy distortion we observe.

The simulation predicts:

(i) NGC 3447 and NGC 3447A belong to the same halo. Moreover, NGC 3447A is a substructure of the same disk that NGC 3447 belongs to. The patchy and lopsided morphology of this system arises from the disk instability induced by the halo gravitational instability itself, enhanced by the interaction. Unrelaxed and/or perturbed halos allow long lasting instabilities in DM dominated disks (Mazzei \& Curir 2001), as in this case. Therefore, the halo gravitational instability drives the disk observed morphology. The system is a "false pair" since NGC 3447A is the result of the disk instability, not a companion.

(ii) The age of NGC $3447 \mathrm{~A} / \mathrm{NGC} 3447$ is $12 \mathrm{Gyr}$, and that of its stellar populations, weighted by $B$-band luminosity, is $1.3 \mathrm{Gyr}$. The stellar mass of this galaxy is $2.2 \times 10^{9} M_{\odot}$, and its total mass is 14.7 times larger than that. 
(iii) The two halos involved in the interaction are separated by about one degree now (Sect. 4.1). Their corresponding galaxies have different luminosities, by $0.5 \mathrm{mag}$ in the $B$-band, the galaxy corresponding to our target, the NGC 3447A/NGC 3447 system, being the brighter one. In Appendix A we discuss the environment of this system, where encounters are highly favoured and there are several dwarf galaxies.

More than 25 years ago, in an invited review, Karachentsev (1989) discussed the incidence of false pairs in his catalogue of 603 isolated pair (Karachentsev 1972, KIG). The incidence of false pairs, a class totally distinct from that of optical pairs, that is, "accidental projection in the line of sight of galaxies that are not in spatial proximity", reaches about $32 \%$ of KIG objects. The false pairs are formed by projection of two members of one group or cluster in the line of sigth that are not gravitationally bound to each other. Following our simulation, the system NGC 3347A/NGC 3347 represents a new class of false pairs, that is, galaxies which appear as pairs but are a single distorted galaxy. Karachentsev warned about the potential danger of an underestimation of the role of false pairs since they gave "anomalously high values of average orbital mass for binary galaxies". The problem reverberates to new large pair surveys. Moreover, the evolution of this DM-dominated system, giving rise to a very common intermediate luminosity galaxy as the result of an encounter with a still far away companion, is a building block in galaxy assembly.

Acknowledgements. We thank the referee for helpful comments improving our manuscript. P.M. and R.R. acknowledge partial financial support by contracts PRIN-INAF-2014-14 "Star formation and evolution in galactic nuclei". We acknowledge the use of public data from the Swift data archive. SDSS calibrated images of the NGC 3347 system were obtained from the data-base developed by Knapen et al. (2014b) (http://vizier.cfa.harvard.edu/ viz-bin/VizieR?-source=J/A+A/569/A91). M.R. acknowledges financial support from grants IN103116 from PAPIIT-UNAM and 253085 from CONACyT. H.P. thanks Laboratoire d'Astrophysique de Marseille for its financial support during his visit in April-July 2017. We acknowledge the use of the HyperLeda database (http://leda.univ-lyon1.fr; Makarov et al. 2014) and the NED, NASA/IPAC Extragalactic Database (http://ned.ipac. caltech.edu), which is operated by the Jet Propulsion Laboratory, California Institute of Technology, under contract with the National Aeronautics and Space Administration.

\section{References}

Amram, P., Balkowski, C., Boulesteix, J., et al. 1996, A\&A, 310 Amram, P., Mendes de Oliveira, C., Plana, H., et al. 2007, A\&A, 471, 753 Bauer, A., Hopkins, A., Gunawardhana, M., et al. 2013, MNRAS, 434, 209 Belokurov, V., Zucker, D. B., Evans, N. W., et al. 2006, ApJ, 647, L111 Bender, R., Surma, P., Döbereiner, S., Möllenhoff, C., \& Madjewsky, R. 1989, A\&A, 217, 35

Bettoni, \& D., Buson, L., Mazzei, P., et al. 2012, MNRAS, 423, 295 Block, D. L., Bournaud, F., Combes, F., et al. 2006, Nature, 443, 832 Bordalo, V., Plana, H., \& Telles, E. 2009, ApJ, 696, 1668

Breeveld, A. A., Curran, P. A., Hoversten, E. A., et al. 2010, MNRAS, 406, 1687 Breeveld, A. A., Landsman, W., Holland, S. T., et al. 2011, in Gamma Ray Bursts 2010, AIP Conf. Proc., 1358, 373

Burstein, D., \& Heiles, C. 1982, AJ, 87, 1165

Buson, L., Bettoni, D., Mazzei, P., \& Galleta, G. 2015, AdAst, E8B Capaccioli, M., Caon, N., \& Rampazzo, R. 1990, MNRAS, 242, 24 Chiosi, C. 2007, EAS Pub. Ser., 27, 25

Christensen, C., Quinn, T., Stinson, G., et al. 2010, ApJ, 717, 121

Christensen, C., Quinn, T., Governato, F., et al. 2012, MNRAS, 425, 3058
Curir, A., \& Mazzei, P. 1999a, New Astron., 4, 1

Curir, A., \& Mazzei, P. 1999b, A\&A, 352, 103

Curir, A., Mazzei, P., \& Murante, G. 2006, A\&A, 447, 453

Curir, A., Mazzei, P., \& Murante, G. 2008, A\&A, 481, 651

Daigle, O., Carignan, C., Hernandez, O., Chemin, L., \& Amram, P. 2006, MNRAS, 368, 1016

de Vaucouleurs, G. 1948, Ann. Astrophys., 11, 247

Dressler, A., \& Shectman, S. A. 1988, AJ, 95, 985

Firth, P., Evstingneeva, E., Jones, B., et al. 2006, MNRAS, 372, 1856

Freeman, K. C. 1970, ApJ, 160, 811

Gordon, K. D., Bailin, J., Engelbracht, C. W., et al. 2006, ApJ, 639, L87

Gonzalez, A., Sivanandam, S., Zabludoff, A., \& Zaritsky, D. 2013, ApJ, 778, 14

Governato, F., Reduzzi, L., \& Rampazzo, R. 1996, MNRAS, 261, 379

Grillmair, C. J. 2006, ApJ, 615, L29

Hoversten, E. A., Gronwall, C., Vanden Berk, D. E., et al. 2011, AJ, 141, 205

Jedrzejewski, R. 1987, MNRAS, 226, 747

Kang, Y., Bianchi, L., \& Rey, S.-C. 2009, ApJ, 703, 614

Karachentsev, I. D. 1972, in Catalogue of isolated pairs of galaxies, Soobshch. Spets. Atrofiz. Obs., 7, 3

Karachentsev, I. D. 1989, in Paired and Interacting Galaxies, NASA Conf. Publ., 3098, 3

Karczewski, O. Ł., Barlow, M. J., Page, M. J., et al. 2013, MNRAS, 431, 2493

Kennicutt, R. C., Jr, 1998, ARA\&A, 36, 189

Knapen, J. H., Erroz-Ferrer, S., Roa, J., et al. 2014a, A\&A, 569, A91

Knapen, J. H., Erroz-Ferrer, S., Roa, J., et al. 2014b, Vizier Online Data Catalog: $\mathrm{J} / \mathrm{A}+\mathrm{A} / 569 / \mathrm{A} 91$

Laval, A., Boulesteix, J., Georgelin, Y. P., Georgelin, Y. M., \& Marcelin, M. 1987, A\&A, 175, 199

Makarov, D., Prugniel, P., Terekhova, N., et al. 2014, A\&A, 570, A13

Marino, A., Bianchi, L., Rampazzo, R., et al. 2010, A\&A, 511, A29

Marino, A., Plana, H., Rampazzo, R., et al. 2013, MNRAS, 428, 476

Marino, A., Mazzei, P., Rampazzo, R., et al. 2013, MNRAS, 459, 2212

Mazzei, P. 2003, Mem. S.A. It, 74, 489

Mazzei, P. 2004, [arXiv: astro-ph/0401509]

Mazzei, P., \& Curir, A. 2001, A\&A, 372, 803

Mazzei, P., \& Curir, A. 2003, ApJ, 591, 784

Mazzei, P., Xu, C., \& De Zotti, G. 1992, A\&A, 256, 45

Mazzei, P., De Zotti, G., \& Xu, C. 1994, ApJ, 422, 81

Mazzei, P., Marino, A., \& Rampazzo, R. 2014a, ApJ, 782, 53

Mazzei, P., Marino, A., Rampazzo, R., Galletta, G., \& Bettoni, D. 2014b, Adv. Space Res., 53, 950

Muñoz-Tuñón, C., Tenorio-Tagle, G., \& Castañeda, H. O. 1996, AJ, 112, 1636

Odenkirchen, M., Grebel, E. K., Rockosi, C. M., et al. 2001, ApJ, 548, L165

Oke, J. B. 1974, ApJS, 27, 21

Pacifici, C., Kassin, A., Weiner, B., Charlot, S., \& Gardner, J. 2013, ApJ, 762, L15

Perea, J., Olmo, A., \& Moles, M. 1990, A\&A, 237, 319

Plana, H., Rampazzo, R., Mazzei, P., et al. 2017, MNRAS, 472, 3074

Poole, T. S., Breeveld, A. A., Page, M. J., et al. 2008, MNRAS, 383, 627

Rampazzo, R., Mazzei, P., Marino, A., et al. 2017, A\&A, 602, A97

Roming, P. W. A., Kennedy, T. E., Mason, K. O., et al. 2005, Space Sci. Rev., 120,95

Roming, P. W. A., Koch, T. S., Oates, S. R., et al. 2009, ApJ, 690, 163

Rosado, M., Langarica, R., Bernal, A., et al. 1995, Rev. Mex. Astron. Astrophys. Conf. Ser., 3, 263

Salpeter, E. E. 1955, ApJ, 121, 161

Sanchez-Cruces, M., Rosado, M., Rodriguez-Gonzalez, A., \& Reyes-Iturbide, J. 2015, ApJ, 799, 231

Schneider, M., Frenk, C., \& Cole, S. 2012, Cosmol. Astropart. Phys., 5, 30

Spavone, M., Iodice, E., Calvi, R., et al. 2009, MNRAS, 393, 317

Tenorio-Tagle, G., Munoz-Tunon, C., \& Cox, D. 1993, ApJ, 418, 767

Trinchieri, G, Marino, A., Mazzei, P., Rampazzo, R., \& Wolter, A. 2012, A\&A, 545, A140

Tully, R., Rizzi, L., Shaya, E., et al. 2009, AJ, 138, 323

Tammann, G. A. 1994, in ESO Conf. and Workshop Proc., eds. G., Meylan, P., Prugniel, Dwarf Galaxies in the Past, 49, 3

Yamanaka, M., Maeda, K., Kawabata, M., et al. 2014, ApJ, 782, L35

Yamauchi, C., Fujishima, S., Ikeda, N., et al. 2011, PASP, 123, 852

Yanny, B., Newberg, H., Grebel, E., et al. 2003, ApJ, 588, 824

Yi, S., Lee, J., Sheen, Y.-K., et al. 2011, ApJS, 195, 22

Warren, M., Quinn, P., Salmon, J., \& Zurek, W. 1992, ApJ, 399, 405

Windhorst, R., Burstein, D., Mathis, D. et al. 1991, ApJ, 380, 362 


\section{Appendix A: The environment of the NGC 3447A/NGC 3447A system: updated vision of the LGC 225 group}

NGC 3447/NGC 3447A belongs to LGG 225 group which occurs in a very crowded region of the Leo Cloud (Fig. A.1, top-left panel). The population of Spirals, which dominate this group, spans a range of ages between a few and $6 \mathrm{Gyr}$ and their total mass ranges from 5 to $35 \times 10^{10} M_{\odot}$ (Marino et al. 2010). We revised the dynamical properties of this group following the recipes in Marino et al. (2013) and Marino et al. (2016). This allows us to improve the membership of the group increasing its number of galaxies from 15 (Marino et al. 2010, including NGC 3447A) to 41 (Table A.2). Figure A.1 (top right panel) highlights the galaxy density and velocity distribution within a box of $4 \times 4 \mathrm{Mpc}^{2}$ centred on this group. Table A.1 points out the updated kinematical and dynamical properties of the group resulting from a non-luminosity weighted analysis of its members based on the recipes in Perea et al. (1990) and Firth et al. (2006), adopting $\mathrm{H}_{0}=75 \mathrm{~km} \mathrm{~s}^{-1} \mathrm{Mpc}^{-1}$. As discussed in these papers, a large scatter in the properties of the group could arise from this approach if we weight by luminosity or not, due to the different distributions of mass and light, and on the adopted wavelength (Marino et al. 2010). Looking at Table A.1, we note a large difference between the virial and projected mass estimates which points towards the presence of anisotropy in the group. This fact, together with the relatively long crossing time $(>0.2$ Hubble time), indicate that this group is not virialized (Firth et al. 2006). This conclusion is further strengthened by the "bubble-plot" (Dressler \& Shectman 1988) in Fig. A.1 (bottom panel) showing a large number of small overlapping bubbles in the region of LGG 225. This means that there are numerous substructures lacking of any global potential field, that is, a larger bubble including several/all of these substructures. Our simulation suggests that one encounter is enough to cause such a morphological, dynamical and photometric perturbation. As a proxi of the role played by galaxy-galaxy interaction, we inspect the group looking for both interacting (pairs) and perturbed members without an obvious companion. Marino et al. (2010) pointed out that LGG 225 group hosts a well known physical pair NGC 3454/3455 (KPG 257, Karachentsev 1972). On the other side, there are several galaxies showing morphological distortions/perturbation. Marino et al. (2010) indicate UGC 6035 (the galaxy nearest to NGC 3447 in projection), UGC 6022, NGC 3370, NGC 3507 NGC 3501, UGC 6083, UGC 6112, UGC 6171. Taking into account that our simulation suggests that NGC 3447/3447A is a single, perturbed system, we conclude that the on-going interactions in the form of classical physical pairs weight for about $5 \%$ of the group members while about 22\% (9/41 including NGC 3447) is the fraction of galaxies with un-ambiguous signatures of perturbations. Roughly 1 out 4 galaxies could have been perturbed by encounters suggesting that the probability of multiple interactions appears low. The NGC 3447/3447A system with its unique morphology, according to our simulation, provides a snapshot of the interaction job in this phase of the group evolution.

\section{A.1. The photometric properties of the group}

Figure A.2, left panel, highlights that its galaxy population is dominated by star-forming galaxies and its ETGs are not passively evolving. Yi et al. (2011) established a colour criterion to classify ETGs according to their UV spectral morphology based on three colour conditions. Passively evolving ETGs would have NUV $-r>5.4$, and FUV $-r>6.6$. These last values indicate the confidence limit of the red sequence in the color magnitude diagrams NUV $-r$ vs. $M_{r}$ and FUV $-r$ vs. $M_{r}$ respectively. ETGs showing UV upturn with no residual star formation have to obey a further condition, FUV-NUV $<0.9$.

The ETGs of this group, namely NGC 3457 and NGC 3522, do not live in the region of passively evolving ETGs, that is, region (c), neither in that showing UV-upturn with no residual star formation, that is region (d) where UV is due to the old star populations. Mazzei et al. (2014a) analysed the multi- $\lambda$ properties of NGC 3457 finding that these are well matched by an encounter, not a merger, between two halos of equal mass and perpendicular spin, as in the case of NGC 3447/NGC 3447A, but with a total mass ten times larger than that. NGC 3522 is likely due to a major merger (mass ratio $1: 1$ ) of initial total mass $4 \times 10^{11} M_{\odot}$. This E galaxy in the $B$-band is one magnitude fainter than NGC 3457. Its evolution in the colour magnitude diagram, NUV $-r$ versus $M_{r}$, shows frequent "rejuvenation" episodes which, of course, cannot change its optical properties but strongly modify the UV ones. Such behaviour occurs in low-luminosity ETGs more than in the brighter ones (Mazzei et al. 2014a). Moreover, Fig. A.2 (right panel) shows that there are no very bright galaxies in the group (i.e. no galaxies with $M_{B}<-20 \mathrm{mag}$ ) and ETGs are not the brightest ones emphasising that luminosity and mass distribution, particularly in the short wavelength range (UV and $B$ bands), are different. This points against the virialization of this group.

The $B$-band absolute magnitudes of Fig. A.2, corrected by galactic extinction following Burstein \& Heiles (1982) as well as the colours, account for the average group distance, $15 \mathrm{Mpc}$ (Marino et al. 2010, their Table 6). Dwarfs, that is, galaxies fainter than $M_{B}=-16 \mathrm{mag}$ (Tammann 1994), are numerous, as expected, in this still not virialized group. The complete analysis of this group deserves, of course, further investigation. We outlined here that the NGC 3447/NGC 3447A system lives in a region where encounters are possible and several late-type galaxies, fainter than that, are present, in agreement with the scenario accounted for by our SPH-CPI simulation. 


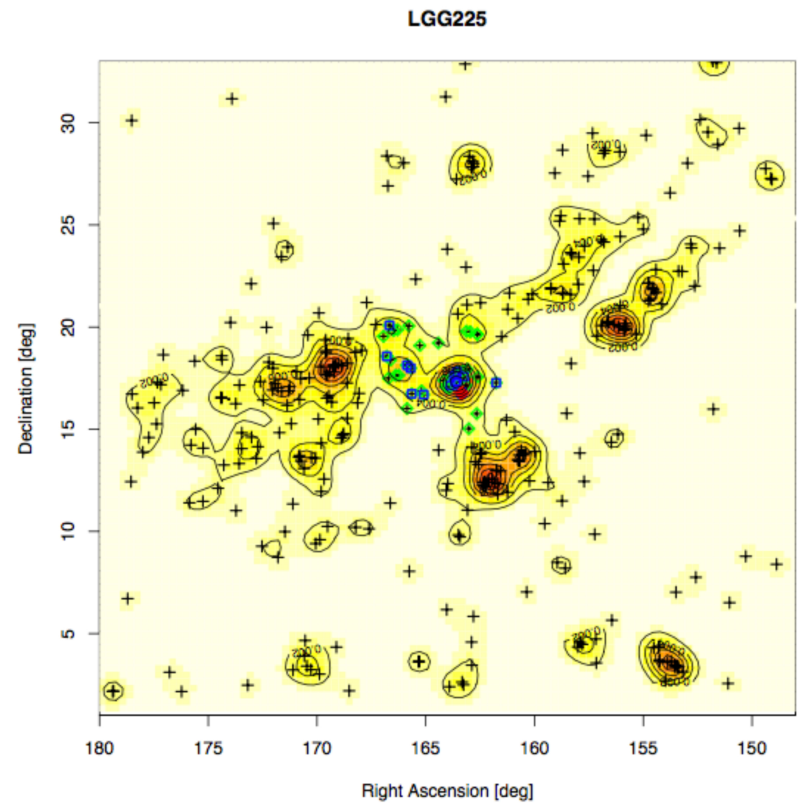

LGG225
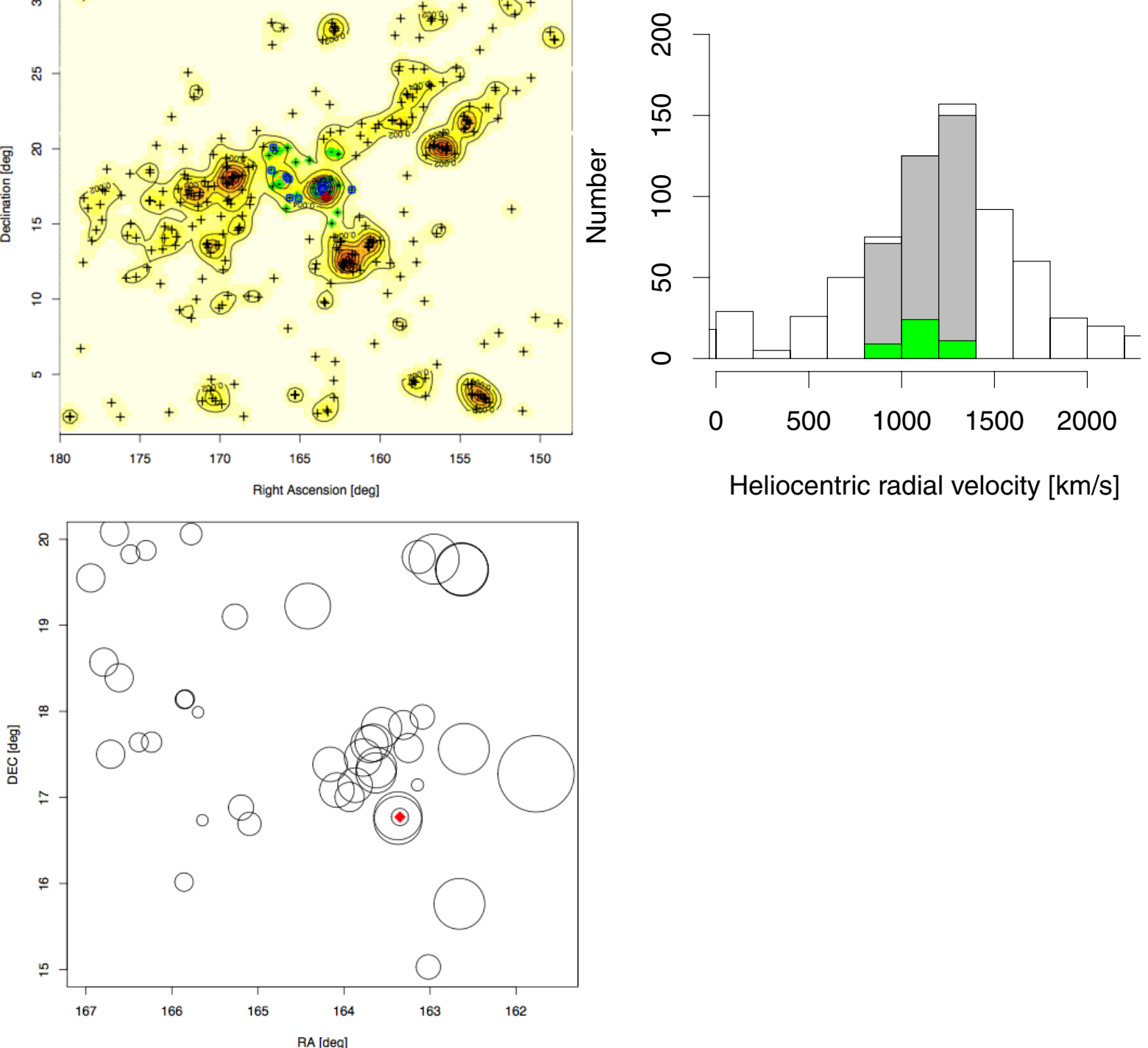

Fig. A.1. Top left: spatial distribution of galaxies (black plus symbols) within a box of $4 \times 4 \mathrm{Mpc}^{2}$ centred on LGG 225 (Marino et al. 2010 , and Table A.1) and with velocity extent, $\sigma$, defined by the dynamical analysis. Blue square symbols show the members of the group in Marino et al. (2010), green diamonds indicate the added members, and the big red filled diamond marks the position of NGC 3447/NGC 3447A. The 2D binned kernel-smoothed number density contours of galaxies fainter than $m_{B}=15.5 \mathrm{mag}$ (crosses) are also shown (red/yellow shaded area). Top right: histogram of the heliocentric radial velocity of all the galaxies within the same box in the left panel accounting for a velocity dispersion $\pm 1000 \mathrm{~km} \mathrm{~s}^{-1}$ around the average velocity of the group (Table A.1). The width of the velocity bins is $200 \mathrm{~km} \mathrm{~s}^{-1}$. The filled (grey) bins show the velocity distribution of galaxies with the same velocity extent as the members of the group; the green bins account for the all the (old+new) members of the group, i.e. galaxies within a box of $1.5 \times 1.5 \mathrm{Mpc}^{2}$ centred on LGG 225 (Table A.2). Bottom: the Dressler \& Shectman (1988) "bubble-plot" based on the ten nearest galaxies. The bubble size is proportional to the squared deviation of the local velocity distribution from the group velocity distribution.

Table A.1. Kinematical and dynamic properties of the updated LGG 225 group.

\begin{tabular}{|c|c|c|c|c|c|c|c|c|c|}
\hline $\begin{array}{l}\text { Group } \\
\text { name }\end{array}$ & $\begin{array}{l}\text { Center } \\
\text { of mass } \\
\text { RA[deg]Dec }\end{array}$ & $\begin{array}{c}V_{\text {group }} \\
{\left[\mathrm{km} \mathrm{s}^{-1}\right]}\end{array}$ & $\begin{array}{c}\text { Velocity } \\
\text { dispersion } \\
{\left[\mathrm{km} \mathrm{s}^{-1}\right]}\end{array}$ & $\begin{array}{c}D \\
{[\mathrm{Mpc}]}\end{array}$ & $\begin{array}{l}\text { Harmonic } \\
\text { radius } \\
{[\mathrm{Mpc}]} \\
\end{array}$ & $\begin{array}{c}\text { Virial } \\
\text { mass } \\
{\left[10^{13} M_{\odot}\right]}\end{array}$ & $\begin{array}{c}\text { Projected } \\
\text { mass } \\
{\left[10^{13} M_{\odot}\right]}\end{array}$ & $\begin{array}{c}\text { Crossing } \\
\text { time } / \mathrm{H}_{0}\end{array}$ & $\begin{array}{c}\text { Group } \\
\text { Luminos. } \\
{\left[10^{11} L_{\odot}\right]}\end{array}$ \\
\hline LGG 225 & $164.45676+17.88861$ & 1116.84 & 122.91 & 14.89 & 0.26 & 0.42 & 1.87 & 0.35 & 0.21 \\
\hline
\end{tabular}



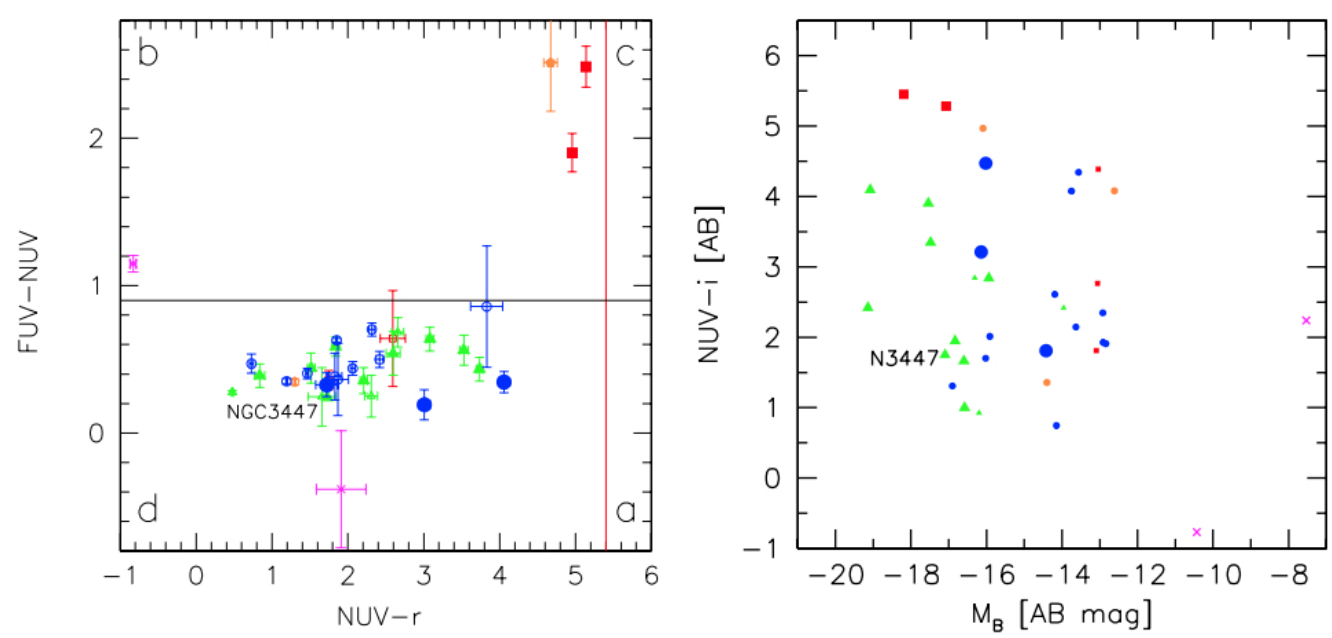

Fig. A.2. Colour-colour diagram, NUV $-r$ vs. FUV-NUV (left panel) and the colour-magnitude diagram, $M_{B}$ vs. NUV $-i$ (right panel), for all the LGG 225 members; (red) squares indicate ETGs $(T \leq-2)$, (orange) diamonds S0s $(-2>T \leq 0)$, (green) triangles Spirals $(T<9)$, (blue) circles show Irregulars $(T \geq 9)$ and magenta crosses are for galaxies without morphological classification in the Hyperleda catalogue; small dots are for new members of this works (Table A.2). Solid lines (left) correspond to the conditions FUV - NUV $<0.9$, that is, UV rising slope, and NUV $-r>5.4$ shows the region of young massive star-free galaxies. These conditions, following the UV classification scheme by Yi et al. (2011), separate passive evolving ETGs (region c) from star forming galaxies (region a), see text. In both panels magnitudes were corrected by galactic extinction following Burstein \& Heiles (1982).

Table A.2. Members of the LGG 225 group.

\begin{tabular}{|c|c|c|c|c|c|c|c|c|}
\hline $\begin{array}{l}\text { Galaxy } \\
\text { name }\end{array}$ & $\begin{array}{l}\text { RA } \\
(\mathrm{J} 2000) \\
{[\mathrm{h}]}\end{array}$ & $\begin{array}{c}\text { Dec } \\
(\mathrm{J} 2000) \\
{[\mathrm{deg}]}\end{array}$ & $\begin{array}{c}\text { Morph } \\
\text { type }\end{array}$ & $\begin{array}{l}\text { Hel. Vel. } \\
{\left[\mathrm{km} \mathrm{s}^{-1}\right]}\end{array}$ & $\begin{array}{c}\log \mathrm{D}_{25} \\
\text { [arcmin] }\end{array}$ & $\begin{array}{r}\operatorname{logr}_{25} \\
{[\mathrm{deg}]}\end{array}$ & $\begin{array}{c}\text { PA } \\
\text { [deg] }\end{array}$ & $\begin{array}{c}B_{T} \\
{[\mathrm{mag}]}\end{array}$ \\
\hline NGC 3370 & 10.78446 & 17.27366 & 5.1 & $1281 \pm 3$ & 1.39 & 0.24 & 146.5 & $12.24 \pm 0.25$ \\
\hline UGC 05945 & 10.84043 & 17.56424 & 9.7 & $1137 \pm 4$ & 0.92 & 0.26 & 94.3 & $15.14 \pm 0.29$ \\
\hline UGC 05947 & 10.84175 & 19.64388 & 10.0 & $1252 \pm 3$ & 1.09 & 0.24 & 25.0 & $14.92 \pm 0.08$ \\
\hline UGC 05948 & 10.84392 & 15.76334 & 10.0 & $1120 \pm 2$ & 1.04 & 0.53 & 35.0 & $17.00 \pm 0.50$ \\
\hline SDSSJ 105148.64+194605.4 & 10.86351 & 19.76823 & 10.0 & $1374 \pm 2$ & 0.56 & 0.30 & 45.8 & $18.34 \pm 0.50$ \\
\hline SDSSJ $105204.79+150149.6$ & 10.86799 & 15.03043 & -5.0 & $828 \pm 38$ & - & - & 5.8 & - \\
\hline PGC 032630 & 10.87261 & 17.93527 & -0.9 & $1304 \pm 4$ & 1.03 & 0.23 & 18.4 & $15.12 \pm 0.32$ \\
\hline UGC 05989 & 10.87551 & 19.79229 & 9.9 & $1138 \pm 2$ & 1.16 & 0.49 & 124.9 & $14.30 \pm 0.29$ \\
\hline SDSSJ $105234.93+170841.8$ & 10.87637 & 17.14494 & 10.0 & $1118 \pm 2$ & 0.49 & 0.12 & 142.2 & $18.25 \pm 0.50$ \\
\hline NGC 3443 & 10.88336 & 17.57442 & 6.6 & $1109 \pm 6$ & 1.25 & 0.30 & 145.7 & $14.58 \pm 0.36$ \\
\hline NGC 3447 & 10.88999 & 16.77242 & 8.8 & $1087 \pm 9$ & 1.53 & 0.24 & 14.3 & $14.47 \pm 0.58$ \\
\hline NGC $3447 \mathrm{~A}$ & 10.89158 & 16.78601 & 9.9 & $1094 \pm 7$ & 1.17 & 0.28 & 107.2 & $15.35 \pm 1.10$ \\
\hline SDSSJ $105329.58+164359.6$ & 10.89155 & 16.73324 & 10.0 & $1074 \pm 2$ & 0.62 & 0.09 & 174.3 & $17.82 \pm 0.50$ \\
\hline UGC 06022 & 10.90427 & 17.81020 & 9.7 & $974 \pm 3$ & 1.03 & 0.24 & 11.1 & $16.76 \pm 0.43$ \\
\hline NGC 3454 & 10.90820 & 17.34398 & 5.4 & $1104 \pm 6$ & 1.38 & 0.66 & 116.2 & $13.71 \pm 0.10$ \\
\hline NGC 3455 & 10.90863 & 17.28473 & 3.1 & $1104 \pm 2$ & 1.36 & 0.20 & 69.7 & $14.30 \pm 0.27$ \\
\hline AGC 208602 & 10.91039 & 17.63500 & - & $1093 \pm 4$ & - & - & - & - \\
\hline NGC 3457 & 10.91350 & 17.62117 & -4.4 & $1159 \pm 5$ & 0.99 & 0.02 & - & $12.98 \pm 0.22$ \\
\hline PGC 1533359 & 10.91852 & 17.46265 & 10.0 & $1167 \pm 1$ & 0.74 & 0.25 & 27.3 & $16.79 \pm 0.50$ \\
\hline UGC 06035 & 10.92473 & 17.14189 & 9.9 & $1073 \pm 2$ & 0.99 & 0.19 & 177.0 & $15.15 \pm 0.32$ \\
\hline PGC 032843 & 10.92901 & 17.00501 & 10.0 & $1138 \pm 2$ & 0.80 & 0.04 & - & $15.26 \pm 0.39$ \\
\hline SDSSJ $105619.93+170505.9$ & 10.93887 & 17.08501 & 10.0 & $961 \pm 1$ & 0.45 & 0.04 & - & $18.25 \pm 0.50$ \\
\hline SDSSJ $105638.66+172301.2$ & 10.94407 & 17.38372 & -5.0 & $948 \pm 1$ & 0.58 & 0.29 & 157.5 & $18.07 \pm 0.50$ \\
\hline SDSSJ $105741.23+191321.1$ & 10.96145 & 19.22252 & 10.0 & $1006 \pm 12$ & - & - & 167.6 & $17.60 \pm 0.50$ \\
\hline UGC 06083 & 11.00661 & 16.69221 & 4.1 & $941 \pm 2$ & 1.13 & 0.92 & 142.5 & $15.19 \pm 0.29$ \\
\hline SDSSJ $110047.14+165255.5$ & 11.01310 & 16.88213 & -5.0 & $1136 \pm 2$ & 0.55 & 0.16 & 101.3 & $18.07 \pm 0.50$ \\
\hline UGC 06095 & 11.01789 & 19.10012 & 9.8 & $1115 \pm 2$ & 1.04 & 0.43 & 5.0 & $16.66 \pm 0.49$ \\
\hline UGC 06112 & 11.04311 & 16.73486 & 7.4 & $1034 \pm 3$ & 1.28 & 0.52 & 120.7 & $14.53 \pm 0.35$ \\
\hline NGC 3501 & 11.04647 & 17.98943 & 5.8 & $1126 \pm 4$ & 1.63 & 0.82 & 27.4 & $13.60 \pm 0.22$ \\
\hline SDSSJ $110306.42+200335.7$ & 11.05181 & 20.06002 & 10.0 & $1284 \pm 5$ & - & - & 72.5 & $21.16 \pm 0.50$ \\
\hline NGC 3507 & 11.05705 & 18.13599 & 3.1 & $976 \pm 7$ & 1.47 & 0.07 & 95.3 & $12.07 \pm 0.51$ \\
\hline PGC 087171 & 11.05732 & 16.01622 & 10.0 & $1230 \pm 3$ & 1.01 & 0.43 & 17.2 & $16.93 \pm 0.50$ \\
\hline SDSSJ $110456.82+173830.5$ & 11.08245 & 17.64178 & 10.0 & $908 \pm 4$ & 0.80 & 0.33 & 113.8 & $17.38 \pm 0.50$ \\
\hline AGC 219617 & 11.08669 & 19.86889 & - & $1199 \pm 6$ & - & - & - & - \\
\hline PGC 033523 & 11.09237 & 17.63961 & 4.5 & $1040 \pm 1$ & 1.19 & 0.40 & 23.8 & $14.82 \pm 0.33$ \\
\hline UGC 06151 & 11.09896 & 19.82534 & 8.7 & $1332 \pm 2$ & 1.08 & 0.02 & - & $14.95 \pm 0.14$ \\
\hline PGC 1558217 & 11.10758 & 18.38999 & 8.0 & $1248 \pm 2$ & 0.61 & 0.15 & 60.9 & $17.18 \pm 0.50$ \\
\hline NGC 3522 & 11.11124 & 20.08561 & -4.9 & $1206 \pm 3$ & 1.07 & 0.28 & 114.7 & $14.07 \pm 0.29$ \\
\hline SDSSJ $110651.09+173002.8$ & 11.11418 & 17.50090 & -2.0 & $949 \pm 71$ & 0.48 & 0.12 & 153.8 & $18.52 \pm 0.50$ \\
\hline UGC 06171 & 11.11949 & 18.57027 & 9.9 & $1203 \pm 1$ & 1.24 & 0.53 & 65.4 & $15.00 \pm 0.28$ \\
\hline UGC 06181 & 11.12962 & 19.54938 & 9.7 & $1170 \pm 3$ & 1.02 & 0.18 & 48.0 & $15.45 \pm 0.32$ \\
\hline
\end{tabular}

